\title{
$\alpha$-Addition von Aminen, Iminen und Hydrazinen an Allenyliden-Komplexe - Bildung von Carben-, Azetidinyliden- und Nitril-Komplexen
}

\author{
Helmut Fischer, Gerhard Roth, David Reindl und Carsten Troll \\ Fakultät für Chemie, Universität Konstanz, Postfach 5560, D-78434 Konstanz 1 (Deutschland)
}

\begin{abstract}
Diarylallenylidene(pentacarbonyl)-chromium and -tungsten complexes, $(\mathrm{CO})_{5} \mathrm{M}=\mathrm{C}=\mathrm{C}=\mathrm{CR}_{2}(\mathrm{M}=\mathrm{W}(\mathbf{1}), \mathrm{Cr}$ (2)), react with amines, imines and hydrazines by addition of the nitrogen nucleophile to the $\mathrm{C}_{\alpha}$ allenylidene atom. With $\mathrm{NEt}_{3}$ the adduct formation is reversible. With secondary and primary amines, imines and hydrazines, the initially formed $N$-ylide complexes react readily further to give carbene, azetidinylidene and nitrile complexes, respectively. For example the reaction of (a) 1 and 2 with $\mathrm{HNEt}_{2}$ or $\mathrm{H}_{2} \mathrm{NPh}$ gives alkenyl(amino)carbene complexes, $\left.(\mathrm{CO})_{5} \mathrm{M}=\mathrm{ClC}(\mathrm{H})=\mathrm{CR}_{2}\right] \mathrm{NR}_{2}^{\prime}(3-5)$; (b) of 1 and 2 with $\mathrm{HN}_{2} \mathrm{CR}_{2}^{\prime}$ gives alkenyl(alkylideneamino)carbene complexes, $(\mathrm{CO})_{5} \mathrm{M}=\mathrm{C}\left[\mathrm{C}(\mathrm{H})=\mathrm{CR}_{2}\right]\left[\mathrm{N}=\mathrm{CR}_{2}^{\prime}\right](6,7) ;(\mathrm{c})$ of 1 with (i $\left.\mathrm{Pr}\right) \mathrm{N}=\mathrm{C}(\mathrm{Ph}) \mathrm{H}$ the azetidinylidene complex 9; (d) of 1 with 1,2-disubstituted hydrazines such as $\mathrm{H}(\mathrm{Me}) \mathrm{NN}(\mathrm{R}) \mathrm{H}(\mathrm{R}=\mathrm{Me}, \mathrm{Ph})$ alkenyl(hydrazino)carbene complexes, $\left.(\mathrm{CO})_{5} \mathrm{~W}=\mathrm{ClC}(\mathrm{H})=\mathrm{CR}_{2}\right][\mathrm{N}(\mathrm{Me}) \mathrm{N}(\mathrm{R}) \mathrm{H}](11)$ and $(\mathrm{e})$ of 1 with $\mathrm{H}_{2} \mathrm{NNR}^{1} \mathrm{R}^{2} \quad\left(\mathrm{R}^{1}=\mathrm{R}^{2}=\mathrm{H}, \quad \mathrm{Me} ; \mathrm{R}^{1}=\mathrm{H}, \quad \mathrm{R}^{2}=\mathrm{Ph}\right)$ alkenyl(amino)carbene complexes (13) and/or acrylnitrile complexes, $(\mathrm{CO})_{5} \mathrm{~W}\left[\mathrm{~N} \equiv \mathrm{C}-\mathrm{C}(\mathrm{H})=\mathrm{CR}_{2}\right](\mathbf{1 2})$. The structures of representative examples of 6,11 and 12 were established by X-ray analyses.
\end{abstract}

\section{Zusammenfassung}

Diarylallenyliden(pentacarbonyl)chrom- und -wolfram-Komplexe, $(\mathrm{CO})_{5} \mathrm{M}=\mathrm{C}=\mathrm{C}=\mathrm{CR}_{2}(\mathrm{M}=\mathrm{W}(\mathbf{1}), \mathrm{Cr}$ (2)), reagieren mit Aminen, Iminen and Hydrazinen unter Addition des Stickstoffnukleophils an das $\mathrm{C}_{\alpha}$-Allenylidenatom. Die Adduktbildung ist mit $\mathrm{NEt}_{3}$ reversibel. Mit sekundären und primären Aminen, Iminen und Hydrazinen reagieren die zunächst gebildeten $N$-Ylidkomplexe rasch weiter zu Carben-, Azetidinyliden- bzw. Nitril-Komplexen. So erhält man z. B. bei der Reaktion von (a) 1 und 2 mit HNEt oder $\mathrm{H}_{2} \mathrm{NPh}$ Alkenyl(amino) carben-Komplexe, $(\mathrm{CO})_{5} \mathrm{M}=\mathrm{C}\left[\mathrm{C}(\mathrm{H})=\mathrm{CR}_{2}\right] \mathrm{NR}_{2}^{\prime}$ (3-5); (b) von 1 und 2 mit $\mathrm{HN}=\mathrm{CR}_{2}^{\prime}$ Alkenyl(alkylidenamino)carben-Komplexe, $(\mathrm{CO})_{5} \mathrm{M}=\mathrm{C}\left[\mathrm{C}(\mathrm{H})=\mathrm{CR}_{2}\right]\left[\mathrm{N}=\mathrm{CR}_{2}^{\prime}\right](6,7)$; (c) von 1 mit ( $\left.{ }^{\mathrm{i} r}\right) \mathrm{N}=\mathrm{C}(\mathrm{Ph}) \mathrm{H}$ den Azetidinyliden-Komplex 9; (d) von 1 mit 1,2-disubstituierten Hydrazinen wie $\mathrm{H}(\mathrm{Me}) \mathrm{NN}(\mathrm{R}) \mathrm{H}(\mathrm{R}=\mathrm{Me}, \mathrm{Ph})$ Alkenyl(hydrazino)carben-Komplexe, $(\mathrm{CO})_{5} \mathrm{~W}=\mathrm{C}$ $\left[C(H)=C_{2}\right][N M e N(R) H](11)$; und (e) von 1 mit $H_{2} N_{N R}^{1} R^{2}\left(R^{1}=R^{2}=H, M e ; R^{1}=H, R^{2}=P h\right.$ ) Alkenyl(amino)carben-Komplexe (13) und/oder Acrylnitril-Komplexe, $(\mathrm{CO})_{5} \mathrm{~W}\left[\mathrm{~N} \equiv \mathrm{C}-\mathrm{C}(\mathrm{H})=\mathrm{CR}_{2}\right](12)$. Die Strukturen repräsentativer Beispiele von 6,11 und 12 wurden durch Röntgenstrukturanalysen gesichert.

\section{Einleitung}

Allenyliden-Komplexe sind seit 1976 bekannt [1,2]. Inzwischen wurde eine Reihe weiterer Synthesen ausgearbeitet, so dass nun Allenyliden-Komplexe der Metalle Chrom, Wolfram, Mangan, Titan, Eisen und
Ruthenium gut zugänglich sind [3]. Entsprechend den mesomeren Grenzstrukturen B und C (Gl. (1)) weisen Allenyliden-Komplexe prinzipiell zwei elektrophile Zentren auf, $\mathrm{C}_{\alpha}$ und $\mathrm{C}_{\gamma}$.

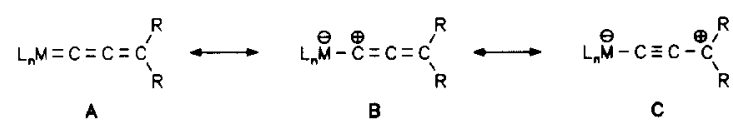

Correspondence to: Prof. Dr. H. Fischer. 


$$
\begin{aligned}
& W(\mathrm{CO})_{6} \underset{-\mathrm{CO}}{\stackrel{\mathrm{h \nu}, \mathrm{THF}}{\longrightarrow}}(\mathrm{CO})_{5} \mathrm{~W}[\mathrm{THF}] \frac{\mathrm{LiC} \equiv \mathrm{C}-\mathrm{CR}_{2} \mathrm{OLi}}{-\mathrm{THF}}\left[(\mathrm{CO})_{5} \mathrm{~W}-\mathrm{C} \equiv \mathrm{C}-\mathrm{CR}_{2} \mathrm{OLi}\right]^{\ominus} \mathrm{Li}^{\oplus} \\
& +\mathrm{COCl}_{2} \mid \begin{array}{l}
-\mathrm{CO}_{2} \\
-2 \mathrm{LiCl}
\end{array}
\end{aligned}
$$

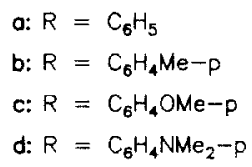

Nach Berke et al. [4] verlaufen die Reaktionen von Allenyliden-Komplexen mit Nukleophilen grenzorbitalkontrolliert. Danach sollen weiche Nukleophile überwiegend am terminalen Allenylidenkohlenstoff $\left(C_{\gamma}\right)$, harte Nuklcophile hingegen hauptsächlich am metallgebundenen $\mathrm{C}$-Atom $\left(\mathrm{C}_{\mathrm{r}}\right)$ angreifen. In Übereinstimmung damit werden neutrale ( $z$. B. Methanol [5]) und anionische (z. B. Alkoholate und Amide [4]) harte Nukleophile im allgemeinen am $\alpha$-Kohlenstoffatom, anionische weiche Nukleophile wie Carbanionen am $\gamma$-Kohlenstoffatom addiert [6]. Ein ambidentes Verhalten zeigt das weiche Thiolatanion: Es wird $\alpha$ und $\gamma$-Addition zugleich beobachtet [4]. Der Ort des Angriffs wird jedoch auch durch die Rückbindungsfähigkeit des $\mathrm{L}_{n} \mathrm{M}$-Fragments beeinflusst. So vermindert das stark rückbindende Fragment $\left[\mathrm{Cl}\left(\mathrm{Ph}_{2} \mathrm{PC}_{2} \mathrm{H}_{4}\right.\right.$ $\left.\left.\mathrm{PPh}_{2}\right)_{2} \mathrm{Ru}\right]^{+}$die Bedeutung der Grenzstruktur $\mathbf{B}$ und dirigiert den Angriff von OMe ${ }^{-}$in die $\gamma$-Position [7].

Neutrale Nukleophile wie tertiäre Phosphane [8] oder Organylisocyanide [9] werden vorzugsweise an das $\mathrm{C}_{\alpha}$-Atom addiert. Am System $\mathrm{Cp}(\mathrm{CO})_{2} \mathrm{Mn}=\mathrm{C}=\mathrm{C}=$ $\mathrm{CPh}_{2} / \mathrm{PPh}_{3}$ konnte dies durch eine Röntgenstrukturanalyse des Addukts bestätigt werden [10]. Für die Reaktionen von $\left(\eta^{5}-\mathrm{C}_{5} \mathrm{H}_{4} \mathrm{Me}\right)(\mathrm{CO})_{2} \mathrm{Mn}=\mathrm{C}=\mathrm{C}=\mathrm{CPh}_{2}$ mit $\mathrm{PPh}_{3}$ bzw. PEt 3 wurde jedoch eine $\gamma$-Addition der Phosphane postuliert [4], wobei das System $\left(\eta^{5}-\mathrm{C}_{5} \mathrm{H}_{4}{ }^{-}\right.$ $\mathrm{Me})(\mathrm{CO})_{2} \mathrm{Mn}=\mathrm{C}=\mathrm{C}=\mathrm{CPh}_{2} / \mathrm{PPh}_{3}$ einen Grenzfall darzustellen scheint: Die $\gamma$-Addition ist reversibel, die Gleichgewichtskonstante ist stark temperatur- und wahrscheinlich auch solvensabhängig. Oberhalb von Raumtemperatur lässt sich im Gleichgewicht auch das $\alpha$-addukt ${ }^{1} \mathrm{H}$-NMR-spektroskopisch nachweisen.

Untersuchungen zum Reaktionsverhalten von Allenyliden-Komplexen gegenüber neutralen Stickstoffbasen wurden bisher kaum bekannt [11*], obwohl Allenyliden-Komplexe als interessante Synthesebausteine für den Aufbau von $N$-Heterocyclen in Betracht $z u$ ziehen sind. Wir berichten nun über die Reaktionen von Diarylallenyliden(pentacarbonyl)chrom- und -wolfram-Komplexen mit verschiedenen N-Nukleophilen wie Aminen, Iminen und Hydrazinen.

\section{Synthese der Diarylallenyliden(pentacarbonyl)- chrom- und -wolfram-Komplexe}

Diarylallenyliden(pentacarbonyl)chrom- und -wolfram-Komplexe lassen sich grundsätzlich über die bereits früher publizierte Folge (a) nukleophile Addition von Dilithiopropargylalkoholaten an $\mathrm{M}(\mathrm{CO})_{6}$, (b) photolytische Decarbonylierung und (c) Desoxygenierung mit Phosgen darstellen [8]. Weniger Nebenprodukte und somit deutlich bessere Ausbeuten liefert jedoch die modifizierte Sequenz (Gl. (2)), in der die beiden Schritte nukleophile Addition (a) und Photolyse (b) vertauscht sind

Der Komplex la wurde bereits beschrieben [8]. Die Stabilität der Verbindungen I nimmt mit steigendem Donoryermögen des Substituenten R. also in der Reihe la-d, deutlich zu. Dic in Lösung tiefblau gefärbten Komplexe la und $\mathbf{1 b}$ sind thermolabil und auch nach chromatographischer Reinigung nur unterhalb $-20^{\circ} \mathrm{C}$ haltbar. 1c (in Pentan blau, in polaren Solventicn blaugrün) ist in kristalliner Form bei Raumtemperatur kurzfristig stabil. la-c wurden daher nur nach obiger Reaktionssequenz "in sim" erzeugt und nach IRspektroskopischer Identifizierung sofort umgesetzt. Id (tiefblau) ist sowohl kristallin als auch in Lösung bei Raumtemperatur stabil.

Dieser Komplex (1d) lässt sich auch auf direktem Weg durch Umsetzung von (CO) $)_{5}$ W $[\mathrm{THF}]$ mit dem entsprechend substituierten Propargylalkohol erhalten (Gl. (3)). Nach der gleichen Methode sind auch die Chrom-Komplexe $2 \mathrm{c}-\mathrm{e}$ (Gl. (3)) in guten bis sehr guten Ausbeuten zugänglich.

Id und $2 \mathbf{c}-\mathbf{e}$ dürften dabei über die intermediäre Bildung eines Akin-Komplexes, Umlagerung zum Vinyliden-Komplex und Wasser-Eliminierung entstehen. Über die gleiche Sequenz sind bereits früher von Selegue Allenylidenruthenium-Komplexe synthetisiert worden [12].

\footnotetext{
* Die Literaturnummer mit einem Sternchen deutet eine Bemerkung in der Literaturliste an.
} 


$$
\begin{aligned}
& (\mathrm{CO})_{5} \mathrm{M}[\mathrm{THF}] \stackrel{\mathrm{HC} \equiv \mathrm{C}-\mathrm{CR}_{2} \mathrm{OH}}{-\mathrm{THF}} \rightarrow\left[(\mathrm{CO})_{5} \mathrm{M}\right. \\
& \text { 1: } M=W \quad \text { c: } \quad C_{2}=C\left(C_{6} H_{4} O M e-p\right)_{2} \\
& \text { 2: } M=C r \quad d: C_{2}=C\left(C_{6} H_{4} N_{M e}-P\right)_{2} \\
& \text { e: } \mathrm{CR}_{2}=\hat{\mathrm{O}}_{\mathrm{c}}^{0} \mathrm{O}
\end{aligned}
$$

3. Reaktionen der Diarylallenyliden-Komplexe mit Stickstoffnukleophilen

\subsection{Reaktionen mit Aminen}

Mit Triethylamin im Überschuss reagiert der Chrom-Komplex 2d unter Addition von $\mathrm{NEt}_{3}$. Die Adduktbildung ist reversibel. Bei einem $\mathrm{NEt}_{3} / \mathbf{2 d}$ Verhältnis von $1 / 1$ liegt das Gleichgewicht noch weitgehend auf der Eduktseite, beim Verhältnis von 10/1 lässt sich nur noch das gelbe Addukt nachweisen (IR $\left.\left(\mathrm{CH}_{2} \mathrm{Cl}_{2}\right): \nu(\mathrm{CO}) 2038 \mathrm{w}, 1928 \mathrm{vs} \mathrm{cm}^{-1}\right)$. Beim Einengen der Lösung und damit Entfernen des Amins wird der blaue Komplex 2d rückgebildet. Aufgrund der vorliegenden spektroskopischen Daten kann nicht entschieden werden, ob sich $\mathrm{NEt}_{3}$ an das $\mathrm{C}_{\alpha}$ - oder an das $\mathrm{C}_{\gamma}$-Atom addiert. Die Befunde mit sekundären Aminen (siehe unten) sprechen jedoch für eine $\alpha$-Addition.

Mit Diethylamin reagieren die Komplexe 1a-d und 2d bereits bei $-70^{\circ} \mathrm{C}$ in $\mathrm{CH}_{2} \mathrm{Cl}_{2}$ innerhalb von Sekunden zu den Alkenyl(amino)carben-Komplexen 3a-d und 4d (Gl. (4)).

Diese sind, wie vergleichbare, auf anderem Weg dargestellte Vertreter dieses Carbenkomplextyps [13], thermisch stabil und zeigen analoge spektroskopische Eigenschaften. Die Reaktionen dürften durch einen unter Ylid-Bildung verlaufenden Angriff von $\mathrm{HNEt}_{2}$ am $\mathrm{C}_{\alpha}$-Atom der Allenyliden-Komplexe eingeleitet

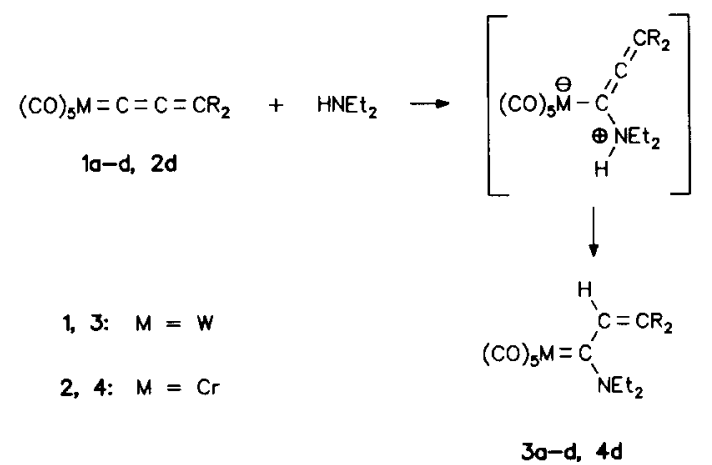

werden. Die nachfolgende Tautomerisierung ergibt dann die Produktkomplexe 3a-d und 4d. Im Fall eines $\gamma$-Angriffs sollten Vinyliden-Komplexe entstehen. Die Umlagerung muss rasch erfolgen, die Ylid-Komplexe konnten bisher nicht nachgewiesen werden.

Ähnlich wie Diethylamin reagieren auch primäre Amine wie Anilin mit den Allenyliden-Komplexen $1 \mathrm{zu}$ Alkenyl(amino)carben-Komplexen (5a，5d; Gl. (5)). Die Umsetzung verläuft wegen der geringeren Nukleophilie von Anilin allerdings langsamer.

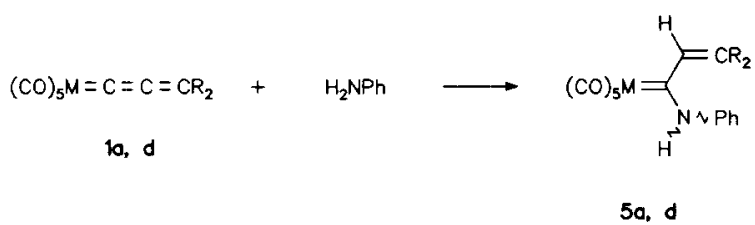

Aus den ${ }^{1} \mathrm{H}$-NMR-Spektren von 5d folgt, dass eines der beiden möglichen Konformationsisomeren bezüglich der partiellen $\mathrm{C}($ Carben $)=\mathrm{N}$-Doppelbindung $(\mathrm{E}$ bzw. Z) bevorzugt gebildet wird. In Übereinstimmung mit den Ergebnissen für andere Aminocarben-Komplexe [14] ist davon auszugehen, dass $5 \mathbf{a}$ und $\mathbf{5 d}$ vorwiegend in der sterisch günstigeren $E$-Konformation vorliegen.

\subsection{Reaktionen mit Iminen}

Die Reaktionen von 1a, 1b und 1d mit $\mathrm{HN}=\mathrm{CPh}_{2}$ bei $-25^{\circ} \mathrm{C}$ bzw. 2e mit $\mathrm{HN}=\mathrm{C}\left({ }^{\mathrm{t}} \mathrm{Bu}\right)$-p-Tolyl bei Raumtemperatur verlaufen analog und führen $\mathrm{zu}$ Alkenyl(alkylidenamino)carben-Komplexen (Gln. (6) und (7)).

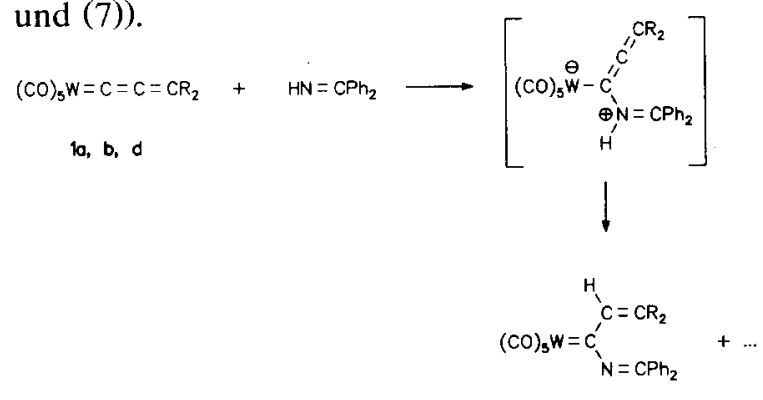


$(\mathrm{CO})_{5} \mathrm{Cr}=\mathrm{C}=\mathrm{C}=\mathrm{CR}_{2}+\mathrm{HN}=\underbrace{\mathrm{t} \mathrm{Bu}}_{\mathrm{C}_{6} \mathrm{H}_{4} \mathrm{Me}-\mathrm{P}} \longrightarrow(\mathrm{CO})_{5} \mathrm{Cr}=\mathrm{C}_{\mathrm{N}=\mathrm{C}\left({ }^{\prime} \mathrm{Bu}\right) \mathrm{C}_{6} \mathrm{H}_{4} \mathrm{CH}_{3}-\mathrm{P}}^{\mathrm{H}}$

$$
\text { 2e } \quad \mathrm{CR}_{2}=\widehat{O O}_{\mathrm{c}}^{\mathrm{o}} \mathrm{XO}
$$

Dieser Carbenkomplextyp war bisher bezüglich der Substituenten nur eingeschränkt zugänglich. Als Nebenprodukt entsteht jeweils durch Substitution des Allenylidenliganden der entsprechende Pentacarbonyl(imin)-Komplex. Ähnlich wie bei den Reaktionen von 1 bzw, 2 mit primären oder sekundären Aminen dürfte zunächst ein bisher nicht nachgewiesener Ylid-Komplex gebildet werden, der dann rasch zu 6 bzw. 7 tautomerisiert. In ihren spektroskopischen Eigenschaften sind $6 \mathbf{a}, 6 \mathbf{b}, 6 \mathbf{d}$ und $7 \mathrm{e}$ denen der kürzlich auf anderem Weg dargestellten ersten Vertreter der Alkenyl(alkylidenamino)carben-Komplexen [15] ähnlich. 6d wurde zusätzlich durch eine Röntgenstrukturanalyse gesichert (Tab. 1, 2 und 3: Abb. 1).

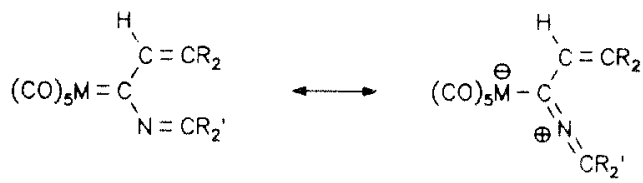

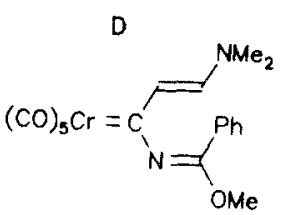

$E$

8

TABELLE 1. Ausgewählte Abstände $(\AA)$ in $\left.(\mathrm{CO})_{5} \mathrm{~W}=\mathrm{C}(\mathrm{N}=\mathrm{CPh})_{2}\right)$ $\left[\mathrm{C}(\mathrm{H})=\mathrm{C}\left(\mathrm{C}_{6} \mathrm{H}_{4} \mathrm{NMe}_{2}-p\right)_{2}\right](6 \mathrm{~d}),(\mathrm{CO})_{5} \mathrm{~W}=\mathrm{C}[\mathrm{N}(\mathrm{Me}) \mathrm{N}(\mathrm{Me}) \mathrm{H}][\mathrm{C}(\mathrm{H})=$

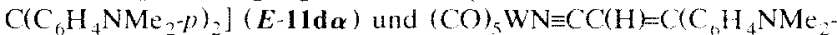
p) ${ }_{2}$ (12d) (Standardabweichungen in Einheiten der letzten signifikanten Stelle in Klammern; für die Atomnumerierung siehe Abbr. 1-3)

\begin{tabular}{llll}
\hline & $6 \mathrm{~d}$ & $E-11 \mathrm{~d} \alpha$ & $12 \mathrm{~d}$ \\
\hline $\mathrm{W}(1)-C(1)$ & $2.033(5)$ & $2.026(8)$ & $2.06(1)$ \\
$\mathrm{W}(1)-C(2)$ & $2.044(5)$ & $2.053(7)$ & $2.06(1)$ \\
$W(1)-C(3)$ & $2.049(5)$ & $2.053(7)$ & $2.03(1)$ \\
$W(1)-C(4)$ & $2.002(5)$ & $2.031(7)$ & $2.64(1)$ \\
$W(1)-C(5)$ & $2.004(4)$ & $2.004(7)$ & $1.96(1)$ \\
$W(1)-C(6)$ & $2.251(3)$ & $2.260(6)$ & \\
$W(1)-N(1)$ & & & $2.18(1)$ \\
$C(6)-C(7)$ & $1.471(5)$ & $1.480(8)$ & $1.42(1)$ \\
$C(6)-N(1) / N(3)]$ & $1.281(4)$ & $1.316(8)$ & \\
$C(7)-C(8)$ & $1.376(5)$ & $1.354(8)$ & $1.34(1)$ \\
$\mathrm{N}(1)-C(9)$ & $1.273(4)$ & & \\
$\mathrm{N}(3)-\mathrm{N}(4)$ & & $1.429(7)$ & \\
$\mathrm{N}(3)-C(25)$ & & $1.485(8)$ & \\
$\mathrm{N}(4)-C(26)$ & & $1.431(10)$ & \\
$\mathrm{N}(1)-C(6)$ & & & $1.14(1)$
\end{tabular}

Anders als der Alkenyl(alkylidenamino)carbenKomplex 8 [15] weist 6d beträchtlichen AzoniaallenylCharakter (E, Gl. (8)) auf.

Der $C(6)-\mathrm{N}(1)-\mathrm{C}(9)$-Winkel ist mit $154.1(4)^{\circ}$ $\left(137.8(3)^{\circ}\right.$ in 8 ) stark aufgeweitet, und die beiden $C$ $\mathrm{N}$-Abstände $(\mathrm{C}(6) \ldots \mathrm{N}(1) \quad 1.281(4), \mathrm{N}(1)-\mathrm{C}(9) 1.273(4)$ $\AA$ ) sind nahezu gleich lang (in 8: C(6)-N(1) $1.348(3)$, $\mathrm{N}(1)-\mathrm{C}(9) 1.263(3) \mathrm{A})$. Der Diederwinkel M-C(6)$N(1)-C(9)$ in $6 \mathbf{d}\left(78.1^{\circ}\right)$ ist allerdings vergleichbar dem

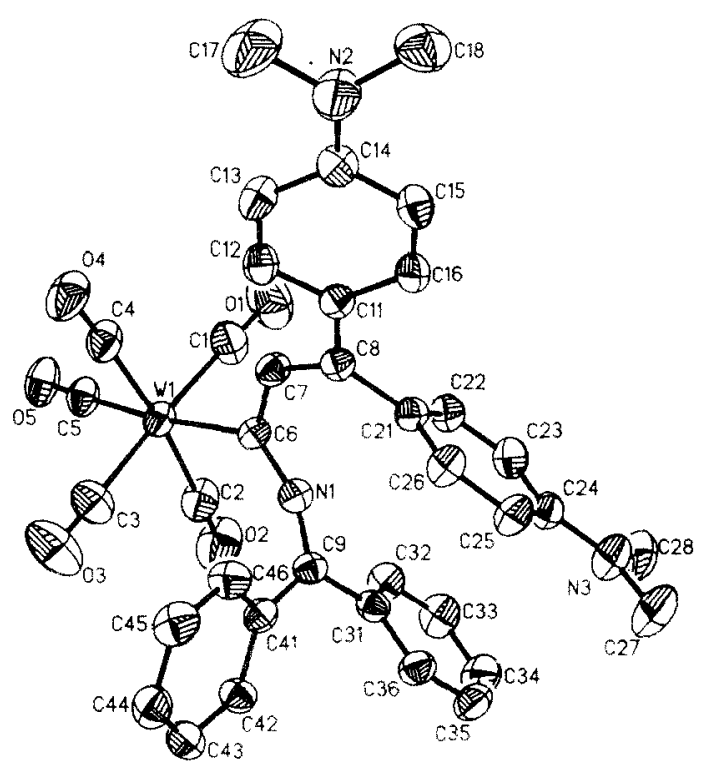

Abb. I. ORTFP-Daritlung von Komplex od im Kristall cohne 1 Atome. Ellipsoide mit 50\%" Aufenthiltswahrscheinlichkeit der Elektronen).

TABELLE 2. Ausgewahlte Winkel (\% in 6d. E-llda und 12d (Standardabweichungen in Einheiten der letzten signifikanten Stelle in Klammern: für die Atommumericrung siche Abbn. 1...3)

\begin{tabular}{|c|c|c|c|}
\hline & $6 d$ & $E-11 \mathrm{~d} \alpha$ & $12 \mathrm{~d}$ \\
\hline$C(5)-W(1)-C(1)$ & $90.702)$ & $94.1(3)$ & $88.0(4)$ \\
\hline$C(5)-W(1)-C(2)$ & $80.112)$ & $80.5(3)$ & $9(0.4(4)$ \\
\hline$C(5)-W(1)-C(3)$ & $95.7(2)$ & $43.7(3)$ & $89.8(4)$ \\
\hline$C(5)-W(1)-C(4)$ & $47.1(2)$ & $\times 2.7(3)$ & $87.8(4)$ \\
\hline$W(1)-(16)-(17)$ & $120.5(2)$ & $116.41+1$ & \\
\hline$C(6)-C(7)-C(x)$ & $128.7(3)$ & $129.1(6)$ & $123.3(9)$ \\
\hline$W(1)-C(6)-N(1) /[N(3)]$ & $110.4(2)$ & $1380(0)$ & \\
\hline$(6)-\mathrm{N}(1)-(6)$ & 154.1141 & & \\
\hline$C(6)-N(3)-C(25)$ & & 124.751 & \\
\hline$(46)-N(3)-N(4)$ & & $12+6(15)$ & \\
\hline$N(3)-N(4)-C(26)$ & & $113.8(5)$ & \\
\hline$W(1)-N(1)-C(6)$ & & & $170.2(8)$ \\
\hline$N(1)-C(6)-C(7)$ & & & $178.1(11)$ \\
\hline
\end{tabular}


TABELLE 3. Atomkoordinaten und äquivalente, isotrope thermische Parameter $\left(\AA^{2}\right)$ für $\mathbf{6 d} ; U_{\mathrm{c} \varphi}=\left(U_{1} U_{2} U_{3}\right)^{1 / 3}$, wobei $U_{i}$ die Eigenwerte der $U_{i j}$-Matrix sind

\begin{tabular}{|c|c|c|c|c|}
\hline Atom & $x$ & $y$ & $z$ & $U_{\mathrm{cu}}$ \\
\hline$W(1)$ & $0.2439(1)$ & $0.0429(1)$ & $0.1909(1)$ & $0.034(1)$ \\
\hline$C(1)$ & $0.1331(5)$ & $0.0932(4)$ & $0.0999(3)$ & $0.056(2)$ \\
\hline$O(1)$ & $0.0729(5)$ & $0.1264(5)$ & $0.0499(3)$ & $0.096(2)$ \\
\hline $\mathrm{C}(2)$ & $0.4083(5)$ & $-0.0017(4)$ & $0.1234(3)$ & $0.053(2)$ \\
\hline$O(2)$ & $0.4993(5)$ & $-0.0334(4)$ & $0.0866(3)$ & $0.089(2)$ \\
\hline$C(3)$ & $0.3611(5)$ & $0.0148(4)$ & $0.2878(3)$ & $0.053(2)$ \\
\hline$O(3)$ & $0.4222(5)$ & $0.0012(4)$ & $0.3436(2)$ & $0.088(2)$ \\
\hline$C(4)$ & $0.0867(5)$ & $0.0700(4)$ & $0.2616(3)$ & $0.047(1)$ \\
\hline $\mathrm{O(4)}$ & $-0.0017(4)$ & $0.0795(3)$ & $0.3037(3)$ & $0.073(1)$ \\
\hline c(5) & $0.1638(4)$ & $-0.1457(4)$ & $0.1533(2)$ & $0.042(1)$ \\
\hline$O(5)$ & $0.1125(4)$ & $-0.2526(3)$ & $0.1308(2)$ & $0.058(1)$ \\
\hline C(6) & $0.3128(3)$ & $0.2607(3)$ & $0.2255(2)$ & $0.032(1)$ \\
\hline$N(1)$ & $0.4386(3)$ & $0.3003(3)$ & $0.2170(2)$ & $0.034(1)$ \\
\hline $\mathrm{C}(9)$ & $0.5690(3)$ & $0.3208(3)$ & $0.2370(2)$ & $0.032(1)$ \\
\hline$C(7)$ & $0.2145(4)$ & $0.3577(3)$ & $0.2562(2)$ & $0.036(1)$ \\
\hline$C(8)$ & $0.2351(3)$ & $0.4937(3)$ & $0.2742(2)$ & $0.031(1)$ \\
\hline$C(11)$ & $0.1370(3)$ & $0.5720(3)$ & $0.3228(2)$ & $0.032(1)$ \\
\hline$C(12)$ & $0.0505(4)$ & $0.5197(3)$ & $0.3721(2)$ & $0.037(1)$ \\
\hline$C(13)$ & $-0.0427(4)$ & $0.5929(4)$ & $0.4165(2)$ & $0.041(1)$ \\
\hline$C(14)$ & $-0.0549(4)$ & $0.7257(4)$ & $0.4139(2)$ & $0.039(1)$ \\
\hline$C(15)$ & $0.0329(4)$ & $0.7800(3)$ & $0.3651(2)$ & $0.040(1)$ \\
\hline$C(16)$ & $0.1258(4)$ & $0.7051(3)$ & $0.3214(2)$ & $0.036(1)$ \\
\hline$N(2)$ & $-0.1470(4)$ & $0.8001(4)$ & $0.4591(3)$ & $0.058(1)$ \\
\hline$C(17)$ & $-0.2406(6)$ & $0.7394(6)$ & $0.5040(4)$ & $0.084(2)$ \\
\hline$C(18)$ & $-0.1762(5)$ & $0.9288(5)$ & $0.4465(3)$ & $0.060(2)$ \\
\hline$C(21)$ & $0.3487(3)$ & $0.5621(3)$ & $0.2414(2)$ & $0.032(1)$ \\
\hline$C(22)$ & $0.3619(4)$ & $0.5340(3)$ & $0.1592(2)$ & $0.037(1)$ \\
\hline$C(23)$ & $0.4673(4)$ & $0.5946(4)$ & $0.1269(2)$ & $0.040(1)$ \\
\hline$C(24)$ & $0.5688(4)$ & $0.6877(4)$ & $0.1750(2)$ & $0.039(1)$ \\
\hline$C(25)$ & $0.5548(4)$ & $0.7171(4)$ & $0.2575(2)$ & $0.044(1)$ \\
\hline$C(26)$ & $0.4463(4)$ & $0.6571(4)$ & $0.2893(2)$ & $0.039(1)$ \\
\hline$N(3)$ & $0.6762(4)$ & $0.7461(4)$ & $0.1433(2)$ & $0.056(1)$ \\
\hline $\mathrm{C}(27)$ & $0.7736(5)$ & $0.8472(6)$ & $0.1929(3)$ & $0.074(2)$ \\
\hline$C(28)$ & $0.6959(6)$ & $0.7088(5)$ & $0.0597(3)$ & $0.063(2)$ \\
\hline$C(31)$ & $0.6670(3)$ & $0.3467(3)$ & $0.1781(2)$ & $0.033(1)$ \\
\hline$C(32)$ & $0.6392(4)$ & $0.2862(4)$ & $0.0974(2)$ & $0.043(1)$ \\
\hline$C(33)$ & $0.7295(5)$ & $0.3085(5)$ & $0.0424(3)$ & $0.054(2)$ \\
\hline$C(34)$ & $0.8511(5)$ & $0.3928(5)$ & $0.0674(3)$ & $0.056(2)$ \\
\hline$C(35)$ & $0.8779(4)$ & $0.4549(5)$ & $0.1467(3)$ & $0.053(2)$ \\
\hline$C(36)$ & $0.7870(4)$ & $0.4325(4)$ & $0.2025(2)$ & $0.041(1)$ \\
\hline$C(41)$ & $0.6207(3)$ & $0.3157(3)$ & $0.3190 \times(2)$ & $0.033(1)$ \\
\hline$C(42)$ & $0.7454(4)$ & $0.2607(4)$ & $0.3322(2)$ & $0.038(1)$ \\
\hline$C(43)$ & $0.7887(4)$ & $0.2502(4)$ & $0.4079(2)$ & $0.044(1)$ \\
\hline$C(44)$ & $0.7096(4)$ & $0.2935(4)$ & $0.4716(2)$ & $0.045(1)$ \\
\hline$C(45)$ & $0.5873(4)$ & $0.3503(4)$ & $0.4590(2)$ & $0.048(1)$ \\
\hline$C(46)$ & $0.5427(4)$ & $0.3608(4)$ & $0.3837(2)$ & $0.043(1)$ \\
\hline
\end{tabular}

in $8\left(81.5^{\circ}\right)$. Ähnliche Winkel werden auch bei anderen Alkylidenaminocarben-Komplexen [16] gefunden. Diese nahezu orthogonale Anordnung von Alkylidenamino- und Carbenebene ermöglicht die Wechselwirkung des freien Elektronenpaars des Alkylidenamino-Stickstoffs mit dem p-Orbital des Carbenkohlenstoffs. Die Abweichung vom idealen $90^{\circ}$ Diederwinkel dürfte sterisch bedingt sein. In Übereinstimmung mit einem beträchtlichen Anteil der Grenz- struktur $\mathbf{E}$ an der Gesamtstruktur ist die W(1)-C(6)Bindung (2.251(3) $\AA$ ) ungewöhnlich lang, und die Resonanz des Carbenkohlenstoffs ist mit $\delta=191.4 \mathrm{im}$ Vergleich zu anderen Carbenkomplexen sehr stark hochfeldverschoben.

Der letzte Schritt der Reaktion von 1 mit $\mathrm{HN}=\mathrm{CPh}_{2}$ - die Umlagerung des postulierten Ylid-Komplexes zu 6-kann durch Substitution des $N$-gebundenen Wasserstoffs durch eine Alkylgruppe blockiert werden. Dann erfolgt formale $(2+2)$-Cycloaddition der $\mathrm{N}=\mathrm{C}$ an die $C_{\alpha}=C_{\beta}$-Bindung: 1a reagiert mit Benzylidenisopropylamin, ${ }^{i} \mathrm{PrN}=\mathrm{C}(\mathrm{Ph}) \mathrm{H}$, im Überschuss langsam zum Azetidinyliden-Komplex 9 (Gl. (9)). Die spektroskopischen Daten von 9 sind vergleichbar denen der röntgenstrukturanalytisch gesicherten Verbindung $\mathbf{1 0}$ [17].

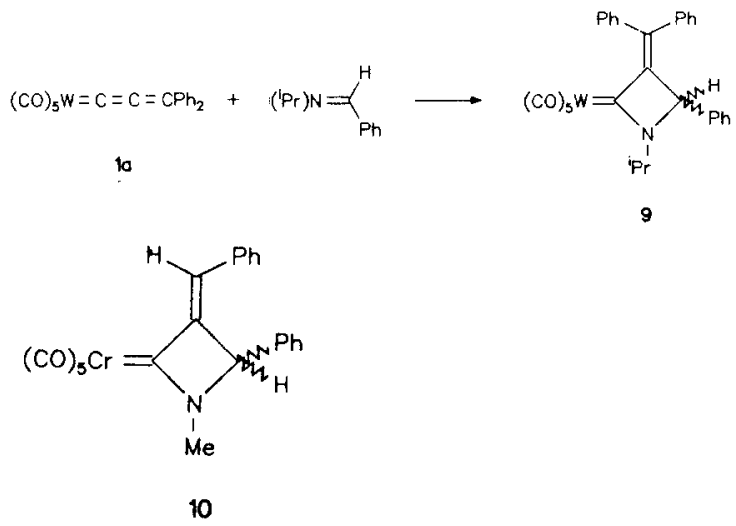

Damit können alternative Formulierungen für 9 wie z. B. als Alkenyl(alkylidenamino)carben-Komplex (vergleichbar 6) oder als Vinyliden-Komplex ausgeschlossen werden. 9 dürfte über die primäre Addition des Imins über den Stickstoff an das $\mathrm{C}_{\alpha}$-Atom von 1a und nachfolgende Cyclisicrung des resultierenden Ylid-Komplexes gebildet werden. Dieser Ablauf entspricht dem der Reaktion von Benzyliden(methyl)amin mit dem kationischen Vinyliden-Komplex $\left\{\mathrm{Cp}\left[\mathrm{P}(\mathrm{OMe})_{3}\right](\mathrm{CO}) \mathrm{Fe}=\mathrm{C}=\mathrm{CMe}_{2}\right\}^{+}[18]$, bei der sich das Primäraddukt isolieren und anschliessend in den Azetidinyliden-Komplex überführen lässt. Im Gegensatz hierzu konnte der Ylid-Komplex bei der Umsetzung von Gl. (9) bisher weder isoliert noch spektroskopisch nachgewiesen werden.

\subsection{Reaktionen mit Hydrazinen}

Der Produkttyp der Reaktionen der AllenylidenKomplexe 1 mit Hydrazinen hängt vom Substitutionsmuster der Hydrazine ab. 1,2-Disubstituierte Hydrazine wie $\mathrm{H}(\mathrm{Me}) \mathrm{NN}(\mathrm{Me}) \mathrm{H}$ und $\mathrm{H}(\mathrm{Me}) \mathrm{NN}(\mathrm{Ph}) \mathrm{H}$ werden im allgemeinen langsam an 1 unter Bildung von Alkenyl(hydrazino)carben-Komplexen addiert. Hydrazin, mono- und 1,1-disubstituierte Hydrazine liefern hingegen hauptsächlich Acrylnitril-Komplexe. Zum Teil 


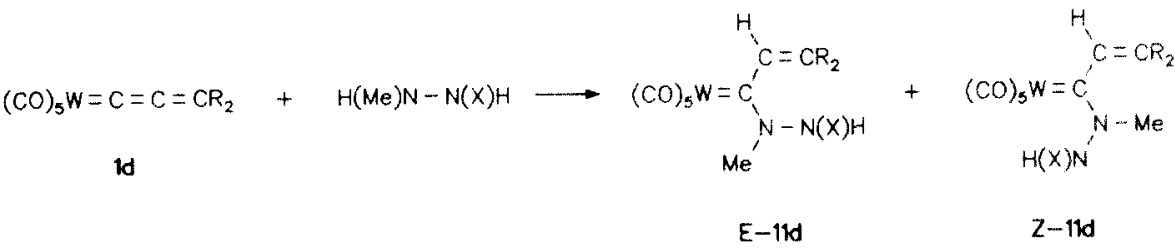

$$
\begin{aligned}
& R=\mathrm{C}_{6} \mathrm{H}_{4} \mathrm{NMe}_{2}-\mathrm{p} \quad \alpha: \mathrm{X}=\mathrm{Me} \\
& \text { B: } X=P h
\end{aligned}
$$

entstehen als Nebenprodukte Alkenyl(amino)carbenKomplexe.

Die Reaktion von 1d mit 1,2-Dimethylhydrazin (Gl. (10)) verläuft deutlich langsamer als dic mit Diethylamin (vgl. Gl. (4)). Dies ist wegen der geringeren Nukleophilie des Hydrazins zu erwarten. Es entsteht ein ca. 1/1-Gemisch zweier isomerer Alkenylhydrazino)carben-Komplexe. Die beiden Isomere unterscheiden sich aufgrund der ${ }^{1} \mathrm{H}-\mathrm{NMR}$-Spektren $\left(\delta\left(\mathrm{N}_{\alpha}\right.\right.$ $\left.\left(\mathrm{CH}_{3}\right)\right)=3.10$ bzw. 3.78) durch die $E$ - und $Z$-Stellung der beiden Substituenten am Carbenkohlenstoffgebundenen Stickstoff (Me und N(MeH) bezüglich der partiellen $C($ Carben $)=N$-Doppelbindung $($ Gl (10): $E-11 d \alpha$ und $Z-11 d \alpha$ ).

Unter vergleichbaren Reaktionsbedingungen ca. 8 mal langsamer als mit $\mathrm{H}(\mathrm{Me}) \mathrm{NN}(\mathrm{Me}) \mathrm{H}$ reagiert $1 \mathrm{~d}$ mit $\mathrm{H}(\mathrm{Me}) \mathrm{NN}(\mathrm{Ph}) \mathrm{H}$. Unter den Produkten lassen sich nur zwei der vier möglichen isomeren Alkenyl(hydrazino)carben-Komplexe nachweisen. Das Aufteten der $\mathrm{N}\left(\right.$ Hydrazino)-CH${ }_{3}$-Resonanzen im 'H-NMR-Spektrum als Singuletts und der Vergleich der Lage mit den entsprechenden Signalen von $11 \mathbf{d} \boldsymbol{\alpha}$ sprechen für die in Gl. (10) für 11d $\beta$ formulierte Struktur (Me mit dem $\mathrm{N}_{\alpha}$-Atom verknüpft) und gegen das alternative Isomer $(\mathrm{CO})_{5} \mathrm{~W}=\mathrm{C}\left[\mathrm{C}(\mathrm{H})=\mathrm{CR}_{2}\right][\mathrm{N}(\mathrm{Ph}) \mathrm{N}(\mathrm{Mc}) \mathrm{H}]$. Dic Reaktion von $1 \mathrm{~d}$ mit $\mathrm{H}(\mathrm{Me}) \mathrm{NN}(\mathrm{Ph}) \mathrm{H}$ ist somit chemospezifisch: Das Hydrazin wird ausschliesslich über die nukleophilere der beiden H(R)N-Gruppen addiert.

Die $E / Z$-Isomerengemische können säulenchromatographisch aufgetrennt werden. Beim Erwärmen bis $70^{\circ} \mathrm{C}$ erfolgt keine Isomerisierung. Die bei Raumtemperatur stabilen Verbindungen 11d $\alpha$ und 11d $\beta$ sind die ersten Vertreter der Klasse der Pentacarbonyl(hydrazinocarben)-Komplexe. Diese waren bisher nicht zugänglich. Frühere Versuche, $(\mathrm{CO})_{5} \mathrm{Cr}=\mathrm{C}(\mathrm{Me})$ OMe durch Hydrazinolyse (OR/ $\mathrm{N}_{2} \mathrm{R}_{3}$-Austausch analog zur Aminolyse) in Hydrazinocarben-Komplexe zu überführen, schlugen fehl [19]. Es wurden vielmehr als Folge einer Umlagerung und unter $\mathrm{N}-\mathrm{N}-\mathrm{Bi}$ ndungsbruch Nitril-Komplexe erhalten (siche unten). Hydrazinocarben-Komplexe des Palladiums, [C], ('Bu$\mathrm{NC}) \mathrm{Pd}=\mathrm{C}\left[\mathrm{N}\left({ }^{\mathrm{t}} \mathrm{Bu}\right) \mathrm{H}\right][\mathrm{NHN}(\mathrm{X}) \mathrm{Y}]$ mit (a) $\mathrm{X}=\mathrm{Ph}, \mathrm{Y}=\mathrm{H}$ und (b) $\mathrm{X}, \mathrm{Y}=\mathrm{C}(\mathrm{H}) \mathrm{Ph}$, sind allerdings bekannt [20].

An $\boldsymbol{E}$-11d $\boldsymbol{\alpha}$ wurde eine Röntgenstrukturanalyse durchgeführt (Tab. 1, 2 und 4: Abb. 2). Das Pentacar-
bonylwolfram-Fragment von $E$-11d $\boldsymbol{\alpha}$ entspricht dem von 6d, die Abstände sind fast identisch. Auch der W(1)-C(6)-Abstand ist mit 2.260(6) $\AA$ sehr lang. Die Atome $\mathrm{C}(6)$ und $\mathrm{N}(3)$ sind trigonal-planar koordiniert (Winkelsumme an $C(6) 359.3^{\circ}$, an $\mathrm{N}(3) 359.6^{\circ}$ ). Dic Carbenebene $[\mathrm{C}(6) / \mathrm{W}(1) / \mathrm{C}(7) / \mathrm{N}(3)]$ und die $\mathrm{N}(3)$. Ebene $[\mathrm{N}(3) / \mathrm{C}(6) / \mathrm{N}(4) / \mathrm{C}(25)]$ sind nahezu koplanar (Diederwinkel $\mathrm{N}(4)-\mathrm{N}(3)-\mathrm{C}(6)-\mathrm{C}(7)-8.2 \%)$. Dadurch

TABELLE 4. Atomkoordinaten und aquivalente. isotrope themi-

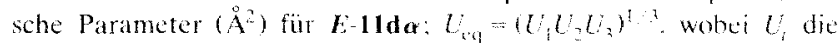

\begin{tabular}{|c|c|c|c|c|}
\hline Atom & $y$ & $y$ & $\therefore$ & $U_{4}$ \\
\hline$W(1)$ & $0.4617(1)$ & $0.2312(1)$ & $0.0618(1)$ & $0.01 .32(1)$ \\
\hline (1) & $0.359366)$ & $0.2539(7)$ & $0.1202(3)$ & $0.047(2)$ \\
\hline $0(1)$ & $0.3030(6)$ & $11.2678(6)$ & $01.1549(3)$ & $0.079(3)$ \\
\hline$((2)$ & $0.5142(6)$ & $11.0173(7)$ & $0.0918(3)$ & $0.0399(2)$ \\
\hline$O(2\}$ & $0.53735)$ & $\cdots 00601165)$ & $0.1050(2)$ & $0.058(2)$ \\
\hline$(13)$ & $0.57076)$ & $11.22(13(6)$ & $11.00183(3)$ & $0.01)+1(2)$ \\
\hline$O(3)$ & $0.6467(5)$ & $0.2149(6)$ & $-11.0208(2)$ & $0.063(2)$ \\
\hline$(4)$ & 0.39176 & $0.3923(7)$ & $11.0217(3)$ & $0.1040(2)$ \\
\hline $0(4)$ & 0.3454159 & $0.4744(5)$ & $-0100133(2)$ & $0.064(2)$ \\
\hline$C(5)$ & $0.3400(6)$ & $(1.1297(7)$ & $0.0144(3)$ & $0.048(2)$ \\
\hline$O(5)$ & $0.2792(5)$ & $(1.67+2(6)$ & $-(1.0129(2)$ & $0.117 \times(2)$ \\
\hline$C(6)$ & 0.5942151 & $0.3+61(6)$ & $011137(2)$ & $0,032(2)$ \\
\hline$(7)$ & $0.7038(5)$ & $0.2774(6)$ & $(1.1267(2)$ & $0.033(2)$ \\
\hline$C(B)$ & $0.7324(5)$ & $0.1823(6)$ & $0.16 .3 \times(2)$ & $0.0130(2)$ \\
\hline c(o) & $0.65650(5)$ & $(0.14336)$ & $0.203,3(2)$ & $0.031(2)$ \\
\hline$(10)$ & $0.6526(5)$ & $0.0073(6)$ & $0.2147(2)$ & $0,03,3(2)$ \\
\hline (111) & $(0.582365)$ & -0.933666 & $0.2572(2)$ & $0.031(2)$ \\
\hline$C(12)$ & $0.5139(5)$ & $10.0500(6)$ & $11.2 \times 12(2)$ & 1).0133(2) \\
\hline$(113)$ & 11.5200651 & $0.1943(6)$ & $0.2055(2)$ & $0.038(2)$ \\
\hline (1) & $0.58 \times 3(5)$ & $0.23,260)$ & $0.2277(2)$ & $0.037(2)$ \\
\hline$N(1)$ & $0.4463(5)$ & $0.017965)$ & $\left(1.31^{4}\right) 4(2)$ & $0.040(2)$ \\
\hline$((15)$ & $0.4573(7)$ & $-0.1160 x)$ & $(1,3413(3)$ & 1).0.58(3) \\
\hline$((16)$ & $0.3815(7)$ & $0.1151(8)$ & $0.3450(3)$ & 1). $06.3(3)$ \\
\hline$(917)$ & $0.843805)$ & $11.1142(6)$ & $0.16+8(2)$ & $0.031(2)$ \\
\hline$C(18)$ & $0.8892(0)$ & $0.0750)(6)$ & 11.119103 & $0.0141(2)$ \\
\hline$C(19)$ & $0.9974(5)$ & $0.022160)$ & $0.1200(3)$ & $0.040(2)$ \\
\hline$C(20)$ & 1.06 .5805 & $0.0(10+0)$ & $0.1678(3)$ & $0.037(2)$ \\
\hline$C(21)$ & $1.0192(5)$ & $0.0423(6)$ & $0.2136(2)$ & $(0.033(2)$ \\
\hline$C(22)$ & $(0.911805$ & 0.190 .3560 & $0.2126(2)$ & $0.032(2)$ \\
\hline$N(2)$ & $1.1744(4)$ & $-0.0490(6)$ & $0.1680(2)$ & $0.043(2)$ \\
\hline$C(23)$ & $1.25011(6)$ & $-0.0417(8)$ & $0.2150(3)$ & $0.1962(3)$ \\
\hline$C(24)$ & $1.2242(6)$ & $-(1.11726(6)$ & $0.120(1) .3)$ & $0.056(3)$ \\
\hline$N(3)$ & $0.5921(4)$ & $0 .+733(5)$ & $0.1280(2)$ & $0,0,30(2)$ \\
\hline$C(25)$ & $0.4917(6)$ & $0.5022(7)$ & $(1.119003)$ & $0.050(2)$ \\
\hline$N(4)$ & $0.6810(5)$ & $0.5386(5)$ & $(1.1504(2)$ & $0.045(2)$ \\
\hline$C(26)$ & $0.7366(7)$ & $0.6+22(8)$ & $0.13 .38(3)$ & $0.067(3)$ \\
\hline
\end{tabular}
Eigenwerte der $l$ Matrix siou 


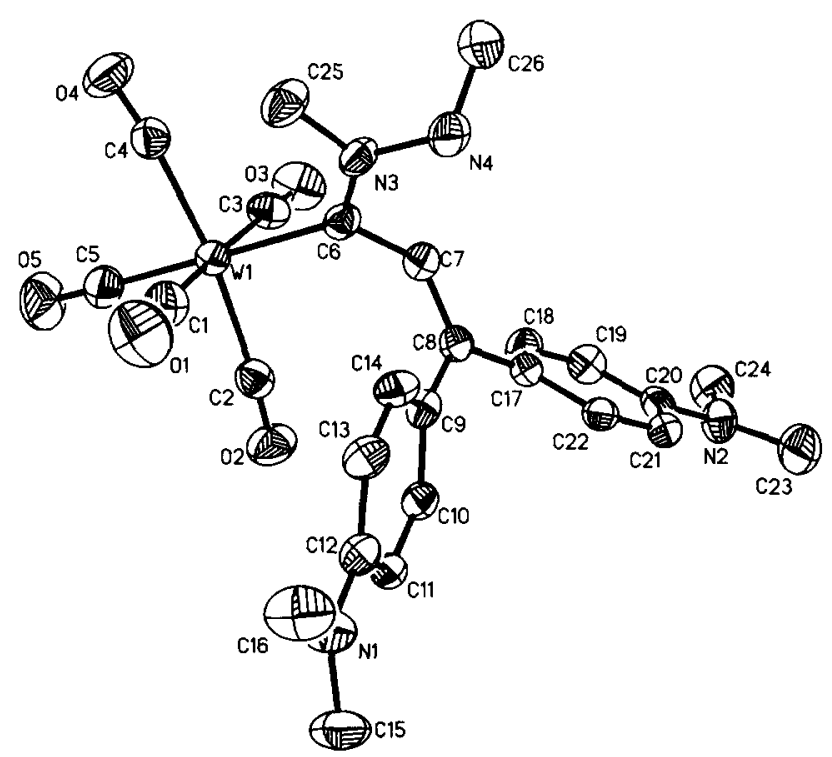

Abb. 2. ORTEP-Darstellung von Komplex $\boldsymbol{E}$-11d $\boldsymbol{\alpha}$ im Kristall (ohne H-Atome, Ellipsoide mit 50\% Aufenthaltswahrscheinlichkeit der Elektronen).

ist eine gute Überlappung des freien Elektronenpaars an N(3) mit dem p-Orbital des Carbenkohlenstoffs gewährleistet. In Übereinstimmung damit ist der C(6)N(3)-Abstand kurz. Der daraus ableitbare starke Doppelbindungscharakter an der $\mathrm{C}(6)-\mathrm{N}(3)$-Bindung erklärt den experimentellen Befund, dass die $E$ - und $Z$-Isomere von 11d thermisch nicht ineinander überführt werden können. Die beiden $\mathrm{N}-\mathrm{CH}_{3}$-Bindungen liegen nicht in einer Ebene, die Vektoren $\mathrm{N}(3)-\mathrm{C}(25)$ und $\mathrm{N}(4)-\mathrm{C}(26)$ sind vielmehr stark gegeneinander verdreht (Diederwinkel C(25)-N(3)$\left.\mathrm{N}(4)-\mathrm{C}(26)-73.6^{\circ}\right)$. Dadurch wird eine ekliptische Stellung zwischen den Elektronenpaaren am $\mathrm{sp}^{2}$-hybridisierten $\mathrm{N}(3)$ - und am $\mathrm{sp}^{3}$-hybridisierten $\mathrm{N}(4)$-Atom vermieden. Zweifellos aus sterischen Gründen sind sowohl die Carben- und die Alkenylebene (Diederwinkel $\left.\mathrm{N}(3)-\mathrm{C}(6)-\mathrm{C}(7)-\mathrm{C}(8) \quad 108.7^{\circ}\right)$ als auch die Alkenylebene und die beiden Arylebenen stark gegeneinander verdreht. Diese Anordnung verhindert im wesentlichen eine denkbare mesomere Wechselwirkung der para-Aminogruppen mit dem Carbenzentrum. Die Abstände $\mathrm{C}(6)-\mathrm{C}(7)$ und $\mathrm{C}(7)-\mathrm{C}(8)$ entsprechen somit auch den Erwartungswerten für $\mathrm{sp}^{2}-\mathrm{sp}^{2}$-Einfach- bzw. $\mathrm{sp}^{2}-\mathrm{sp}^{2}$-Doppelbindungen.

Im Gegensatz zu den Reaktionen von 1d mit 1,2-disubstituierten Hydrazinen werden bei den Umsetzun-

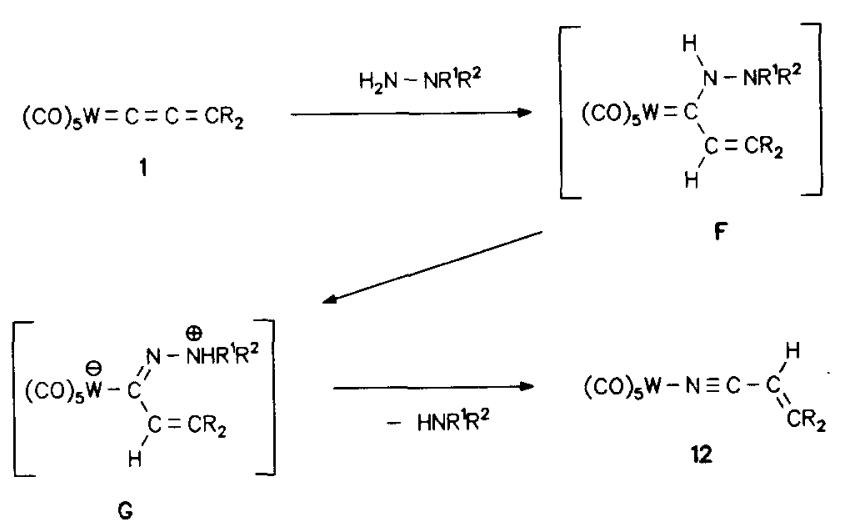

Schema 1.

gen von 1a, 1c und 1d mit Hydrazin, Phenylhydrazin bzw. 1,1-Dimethylhydrazin keine isolierbaren $\mathrm{Hy}$ drazinocarben-Komplexe erhalten. Es entstehen vielmehr die Acrylnitril-Komplexe 12a, 12c bzw. 12d und zusätzlich-mit 1,1-Dimethylhydrazin als Substrat -Alkenyl(dimethylamino)carben-Komplexe (13a, d) (Gl. (11)), 12a und 13a bzw. 12d und 13d werden dabei in annähernd äquimolaren Mengen gebildet. Bei der Umsetzung von $\mathbf{1 b}$ mit Benzophenonhydrazon tritt ausser dem Acrylnitril-Komplex 12b die Verbindung $(\mathrm{CO})_{5} \mathrm{~W}\left[\mathrm{~N}(\mathrm{H})=\mathrm{CPh}_{2}\right]$ als Nebenprodukt auf.

Die Bildung der Produkte 12 und 13 lässt sich durch die in Schema 1 skizzierte Reaktionsfolge verstehen. Zunächst wird durch nukleophile Addition des Hydrazins (über das nicht-substituierte $\mathrm{N}$-Atom) am $\mathrm{C}_{\alpha^{-}}$ Atom von 1 und nachfolgende Tautomerisierung ein Alkenyl(hydrazino)carben-Komplex gebildet. Dieser ist aufgrund der acidifizierenden Wirkung des $(\mathrm{CO})_{5} \mathrm{M}$ Fragments nicht stabil ( $\alpha$-CH-Bindungen in Alkylcarben-Komplexen sind acid; z. B. ist der $\mathrm{p} K_{\mathrm{a}}$-Wert von $(\mathrm{CO})_{5} \mathrm{Cr}=\mathrm{C}(\mathrm{Me}) \mathrm{OMe}$ vergleichbar mit dem von $p$ Cyanophenol (in Wasser: $\mathrm{p} K_{\mathrm{a}}=8$ [21])). F dürfte daher rasch in einer metallorganischen Variante der Beckmann-Umlagerung zu 12 und $\mathrm{HNR}^{1} \mathrm{R}^{2}$ weiterreagieren (Schema 1). Durch entweder intramolekulare Isomerisierung oder $\alpha$-N-Deprotonierung $/ \beta$-N-Protonierung entsteht die dipolare Verbindung $\mathbf{G}$, die sich dann unter $\mathrm{HNR}^{1} \mathrm{R}^{2}$-Eliminierung und 1,2-Wanderung des $(\mathrm{CO})_{5} \mathrm{M}$-Fragments zu 12 stabilisiert. Über eine ähnliche Folge wurde bereits früher die Bildung von Nitril-Komplexen bei der Reaktion von Hydrazinen mit Carben- [19] bzw. Vinyliden-Komplexen [22] erklärt.

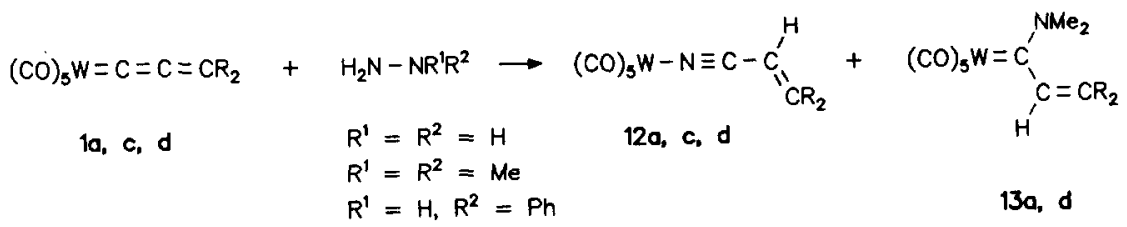$$
R^{1}=R^{2}=M e
$$$$
R^{1}=H_{1} R^{2}=P h
$$

$130, d$ 
TABELLE 5. Atomkoordinaten und itquivalente. isotrope thermi

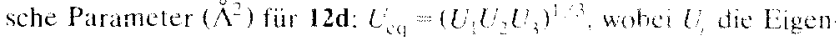
werte der $\ell_{1,}$-Matrix sind

\begin{tabular}{|c|c|c|c|c|}
\hline Atom & $x$ & $y$ & $z$ & $\zeta_{44}$ \\
\hline$W(1)$ & $0.3529(1)$ & $0.4233(1)$ & $0.0131011)$ & $0.0132(1)$ \\
\hline$C(1)$ & $0.4363(5)$ & $0.3230(16)$ & $(0.0) 26,5(3)$ & $0,(1)+5(4)$ \\
\hline$O(1)$ & 0.4831131 & $0.2741(14)$ & $0.025102)$ & $10.067(3)$ \\
\hline$(2)$ & 0.382244 & $10.6934(15)$ & $0.0145(3)$ & $0,033(3)$ \\
\hline$O(2)$ & $(1.3977(3)$ & $0.8450(11)$ & $0.10072(2)$ & $0,055(3)$ \\
\hline$(3)$ & $0.273165)$ & $0.5395(14)$ & $0.036763)$ & $(1,1)+3(4)$ \\
\hline$O(3)$ & $0.2292(3)$ & $0.6101011)$ & $0.14+10(3)$ & $0 .(10.3(3)$ \\
\hline$C(4)$ & 0.323765 & 0.1504106 & $0.01403(3)$ & $10,042(3)$ \\
\hline$O(4)$ & 0.3075011 & $0.0185(11)$ & $(1,16027(2)$ & $0.05 \times(3)$ \\
\hline$C(5)$ & 0.774451 & $0.48014(4)$ & $0118 \times 58(3)$ & $11,14 \times(4)$ \\
\hline $0(5)$ & $0.385764)$ & $0.511 .3(12)$ & $0.123302)$ & $1006,8(3)$ \\
\hline$N(1)$ & $0.3323(3)$ & $0.3460(12)$ & $\cdots 010329(2)$ & $19.1) 40(3)$ \\
\hline$(6)$ & $\{1.3242(4)$ & $0.3123(14)$ & $-0,060693)$ & $00038(3)$ \\
\hline$C(7)$ & $0.314 .3(4)$ & $0.2627(15)$ & $-0.103903)$ & $0,039(3)$ \\
\hline$C(8)$ & $0.3+15(4)$ & $0.3500(14)$ & $-0.1 .388(3)$ & $0.1130(3)$ \\
\hline$C(11)$ & 0.377744 & $0.5257(1.6)$ & $0.1324(3)$ & $0.013+(3)$ \\
\hline$C(12)$ & $0.4286(5)$ & $0.540+117)$ & $0.1518(3)$ & $10.0554)$ \\
\hline$C(13)$ & $0.465 \times(5)$ & $0.70 .39(18)$ & $--(1.1+14.2(3)$ & $0,059(4)$ \\
\hline$C(14)$ & $0.4545(5)$ & $0.84+5(10)$ & $-01.116103)$ & $0.044(3)$ \\
\hline$C(15)$ & $0.402 .45)$ & $0.8250115)$ & $-0.0966(3)$ & $10.1144+1)$ \\
\hline$C(16)$ & $0.3650(+)$ & $0.6680(14)$ & $\cdots(1) .1043(3)$ & $0.038(3)$ \\
\hline$N(2)$ & $0.4903(5)$ & $1.9(12+114)$ & $-0.1600(3)$ & $0,06,3(4)$ \\
\hline$(718)$ & $0.477605)$ & $1.1445(16)$ & $-(1.677844)$ & $0007015)$ \\
\hline$((17)$ & $0.544360)$ & $1,(11+4(22)$ & $0.1268(5)$ & $0,1993(7)$ \\
\hline$C(21)$ & $(0.3349(4)$ & $0.260,7(1.4)$ & (1) 180803$)$ & $19.1034(3)$ \\
\hline$(C(22)$ & $0.33345)$ & $0.06701(7)$ & $-(1.1871(3)$ & $0,0.49(4)$ \\
\hline$(23)$ & 0.927465 & $-001301(14)$ & $-(1.225413)$ & $0,1040(4)$ \\
\hline$C(24)$ & $0.32(131+1)$ & $0.10,9415\}$ & $-11.200063)$ & $\{0.114 .3(3)$ \\
\hline$C(25)$ & $0.3214(5)$ & $0.3055(14)$ & $\cdots-(0.25+4(3)$ & $0.056(4)$ \\
\hline$(126)$ & $0.3292(5)$ & $0.3807014)$ & $-0.0155(3)$ & $0.147(4)$ \\
\hline$N(3)$ & $0.314515)$ & $0,19 \times 2(13)$ & $-(1.299463)$ & $10.1157(4)$ \\
\hline$C(27)$ & $0.320200)$ & $\cdots 11.18011(17)$ & $\cdots(1.39152(3)$ & $0.16765)$ \\
\hline$(.2 N)$ & $0.301 .3(6)$ & $0.1468(19)$ & $-0(1.3353(3)$ & $0.076(6)$ \\
\hline
\end{tabular}

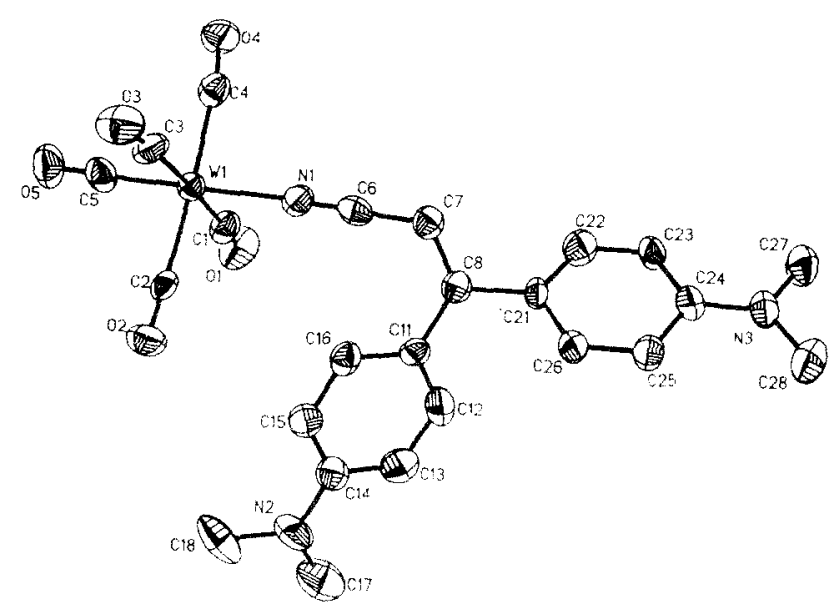

Abb. 3. ORTry-Darstellung von Komplex $12 \mathrm{~d}$ im Kristall Gohne IIAtome. Ellipsoide nit $50 \%$ Aufenthahswahrseheindichkejt der Elektronen).
Wihrend die erste Reaktion $\left(1+\mathrm{H}_{2} \mathrm{NNR}^{\prime} \mathrm{R}^{2} \rightarrow \mathbf{F}\right)$ nur langsam abläuft, sind die weiteren Schritte $\mathbf{F} \rightarrow \mathbf{G}$ $\rightarrow \mathbf{1 2}+$ HNR' $^{2}$ schnell. Weder $\mathbf{F}$, noch $\mathbf{G}$ lassen sich nachweisen. Bei genügend grosser Nukleophilic des freigesetzten Amins ( $z$. B. HNMe $)_{2}$ vermag dieses als Konkurrenzsubstrat zu fungieren und sich an 1 unter Bildung von Alkenylaminokarben-Komplexen (13) anzulagern.

Im Fall von 1,1-Dimethylhydrazin wäre zwar zu erwarten, dass das substituierte Stickstoffatom des $\mathrm{Hy}$ drazins am Allenyliden-Komplex angreift. diese Addition ist jedoch teversibel (vgl. die Reaktion von $2 \mathbf{2 d}$ mit NEt, (3./)). Die Addition üher die NH,-Gruppe ist zwat aufgrund der geringeren Nukleophilie weniger hegünstigt, ist jedech im Sinne von Gl. 11 produktiv und fiihrt irreversibel zu den Produkten 12 und HNME.

Von 12d wurde cine Röntgenstrukturanalyse angefertigt (Tab. 1, 2 und 5; Abb. 3). Das nahezu lincare W(1)-N(1)-C(6)-Fragment belegt die bei NitrilKomplexen bevorzugte end-on-Koordination. Die beobachteten Bindungslängen cntsprechen weitgehend denen für freies Acrylnitril [2.3]. Autfallend ist der starke trans-Effekt. Die W-CO, -Bindung (1.96(1) A) ist signilikant kürer als das Mittel der $\mathrm{W}_{-} \mathrm{CO}$. Bindungen $(2.115 \AA$ A $)$

\section{Fazit}

Alle Produkte der Reaktionen von Allenyliden(pentacarbonyl)-Komplexen mit $\mathrm{N}$-Nukleophilen lassen sich über einen einleitenden Angriff am $C_{i n}$-Atom der Komplexe crklären. Hinweise für cine $\gamma$-Addition gibt es nicht. Dicse Befunde stehen im Einklang mit dem von Berke at al. [4] vorgeschlagenen Reaktivitätsmodell.

Präparativ von Bedeutung sind vor allem dic zu sonst nur schwer oder nicht zugänglichen Produkten führenden Umsetzungen mit Iminen und Hydrazinen. Dabei zeigt sich, dass Pentacarbonyl(hydrazinocarben)-Komplexe offensichtich nur dann stabil und isolierbar sind. wenn Folgereaktionen durch Substitu-

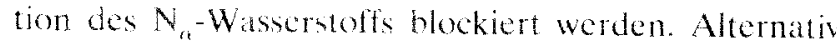
düfte sich cine Stabilisierung auch durch dic Verwendung elcktronenreicher. stark rüchbindender $\mathrm{L}_{\text {. }} \mathrm{M}$ Fragmente erreichen lassen. Fraglich ist jedoch. ob dann die entsprechenden Allenyliden-Komplexe noch Hydrazine 711 Hydrazinocarben-Komplexen addieren (vgl. hiersuauch ! +!).

\section{Experimenteller Teil}

Alle Arbeiten wurden unter Ausschluss von Luft und Feuchtigkeit in N-Atmosphäre durchgeführt. Die 
verwendeten Lösungsmittel waren getrocknet ( $\mathrm{Na}$, $\mathrm{CaH}_{2}$ ) und ebenso wie das zur Chromatographie verwendete Kieselgel (Fa. Baker Nr. 70241) mit Stickstoff gesättigt. Die Ausbeuten beziehen sich auf analysenreine Substanzen und sind nicht optimiert. Die eingesetzten Propargylalkohole [24], Benzophenonimin [25], ${ }^{t}$ Butyl-p-tolylmethylenamin [26], Benzylidenisopropylamin [27] und 1-Methyl-2-phenylhydrazin [28] wurden nach bekannten Vorschriften dargestellt. Alle anderen Chemikalien waren handelsüblich (Fluka, Merck). Die NMR-Resonanzen sind auf TMS ( $\left.{ }^{1} \mathrm{H}-\mathrm{NMR}\right)$ oder das verwendete Lösungsmittel ( $\left.{ }^{13} \mathrm{C}-\mathrm{NMR}\right)$ bezogen.

\subsection{Darstellung von $\left.(\mathrm{CO})_{5} \mathrm{~W} / \mathrm{THF}\right]$ und $(\mathrm{CO})_{5} \mathrm{Cr}[\mathrm{THF}]$}

Eine Lösung von $20 \mathrm{mmol}(7.04 \mathrm{~g}) \mathrm{W}(\mathrm{CO})_{6}$ in 320 $\mathrm{ml} \mathrm{THF} \mathrm{bzw.} \mathrm{von} 20 \mathrm{mmol}(4.40 \mathrm{~g}) \mathrm{Cr}(\mathrm{CO})_{6}$ in $250 \mathrm{ml}$ THF wird jeweils in einer $\mathrm{N}_{2}$-gefluteten Falling-FilmApparatur (Normag-Photoreaktor A9356) unter $\mathrm{N}_{2}-$ Durchfluss bei $10^{\circ} \mathrm{C}$ bestrahlt (Hg-Hochdrucklampe, $150 \mathrm{~W})$. Nach $c a .2 \mathrm{~h}\left[\mathrm{~W}(\mathrm{CO})_{6}\right]$ bis $4 \mathrm{~h}\left[\mathrm{Cr}(\mathrm{CO})_{6}\right]$ ist aufgrund des IR-Spektrums das $\mathrm{M}(\mathrm{CO})_{6}$ fast quantitativ umgesetzt. Die gelb-orange gefärbten Lösungen von $(\mathrm{CO})_{5} \mathrm{~W}[\mathrm{THF}]$ und $(\mathrm{CO})_{5} \mathrm{Cr}[\mathrm{THF}]$ sind bei $-30^{\circ} \mathrm{C}$ mehrere Wochen unverändert haltbar. $(\mathrm{CO})_{5} \mathrm{~W}[\mathrm{THF}]$ : IR (THF): $\nu$ (CO) 2074vw, 1929vs, $1891 \mathrm{w} \mathrm{cm}^{-1}$. $(\mathrm{CO})_{5} \mathrm{Cr}[\mathrm{THF}]:$ IR (THF): $\nu(\mathrm{CO}) 2072 \mathrm{vw}, 1938 \mathrm{vs}$, $1886 \mathrm{~m} \mathrm{~cm}^{-1}$.

\subsection{Darstellung der Diarylallenyliden(pentacarbonyl)- wolfram- und -chrom-Komplexe $1 \mathbf{a}-\boldsymbol{d}$ und $2 \mathrm{c}-\mathrm{e}$}

\subsubsection{Allgemeine Vorschrift für die Synthese von la-d}

Eine Lösung von $5 \mathrm{mmol}$ des jeweiligen Propargylalkohols (1a: $1.04 \mathrm{~g}$ 1,1-Diphenylprop-2-in-1-ol, 1b: 1.18 g 1,1-Bis( $p$-tolyl)prop-2-in-1-ol, 1c: $1.34 \mathrm{~g} 1,1$-Bis $(p$ methoxyphenyl)prop-2-in-1-ol, 1d: $1.47 \mathrm{~g} \mathrm{1,1-Bis(} p$-dimethylaminophenyl)prop-2-in-1-ol) in $25 \mathrm{ml} \mathrm{Et}_{2} \mathrm{O}$ wird bei $-75^{\circ} \mathrm{C}$ mit $10 \mathrm{mmol}(6.25 \mathrm{ml}$ einer $1.6 \mathrm{M}$ Lösung in n-Hexan) n-Butyllithium versetzt. Beim Erwärmen auf Raumtemp. fallen die Dilithiumpropargylalkoholate in Form eines weissen Niederschlags aus. Zur jeweiligen Suspension werden bei Raumtemp. $5 \mathrm{mmol}(80 \mathrm{ml}$ einer $6.25 \cdot 10^{-2} \mathrm{M}$ Lösung in THF) $(\mathrm{CO})_{5} \mathrm{~W}[\mathrm{THF}]$ gegeben und $3 \mathrm{~h}$ gerührt. Die rotbraunen Lösungen werden im Vakuum zur Trockne gebracht und der Rückstand bei $-75^{\circ} \mathrm{C}$ in $25 \mathrm{ml}$ auf $-90^{\circ} \mathrm{C}$ vorgekühltem $\mathrm{CH}_{2} \mathrm{Cl}_{2}$ aufgenommen. Nach Zugabe von $2.6 \mathrm{ml}$ Phosgenlösung (1.93 $\mathrm{M}$ in Toluol, $5 \mathrm{mmol}$ ) lässt man das Reaktionsgemisch unter Farbumschlag nach blau (1a, 1b, 1d) bzw. blaugrün (1c) auf $-20^{\circ} \mathrm{C}$ erwärmen. Die Produkte werden in unterschiedlicher Weise aufgearbeitet:

Pentacarbonyl(diphenylallenyliden)wolfram (1a). 1a wird nicht isoliert sondern direkt umgesetzt. Die Identifizierung erfolgt IR-spektroskopisch (n-Pentan): $\nu(\mathrm{CO})$ $2070 \mathrm{w}, 1991 \mathrm{~m}, 1977 \mathrm{vs} \mathrm{cm}{ }^{-1} ; \nu(C C C) 1920 \mathrm{~m} \mathrm{~cm}^{-1}$.

/Bis(p-tolyl)allenyliden/(pentacarbonyl)wolfram (Ib). 1b wird nicht isoliert und direkt umgesetzt. Die Identifizierung erfolgt IR-spektroskopisch (n-Pentan): $\nu(\mathrm{CO})$ $2075 \mathrm{w}, 1961 \mathrm{~m}, 1935 \mathrm{vs} \mathrm{cm}^{-1} ; \nu$ (CCC) $1913 \mathrm{~m} \mathrm{~cm}^{-1}$.

[Bis (p-methoxyphenyl)allenyliden] (pentacarbonyl)wolfram (Ic). 1c kann durch Chromatographie an $\mathrm{Al}_{2} \mathrm{O}_{3}$ (Akt.-Stufe 3-4) bei $-50^{\circ} \mathrm{C}$ gereinigt werden: Elution zunächst mit Pentan, anschliessend mit Pentan $/ \mathrm{Et}_{2} \mathrm{O}$ (aufsteigend polar bis 3/1). Nach einem schwach gelben Vorlauf wird 1c als türkis-farbene Zone abgetrennt. Nach Entfernen des Lösungsmittels im Vakuum bleibt ein schwarzes Pulver zurück. Ausb. $0.31 \mathrm{~g} \mathrm{(11 \%}$ bez. auf $\left.\mathrm{W}(\mathrm{CO})_{6}\right)$. IR $\left(\mathrm{Et}_{2} \mathrm{O}\right): \nu(\mathrm{CO}) 2072 \mathrm{w}, 1966 \mathrm{~m}$, $1952 \mathrm{vs} \mathrm{cm}^{-1} ; \nu(\mathrm{CCC}) 1926 \mathrm{~m} \mathrm{~cm}^{-1} .{ }^{1} \mathrm{H}-\mathrm{NMR}\left(\mathrm{CDCl}_{3}\right.$, $\left.-10^{\circ} \mathrm{C}\right): \delta=3.93\left(\mathrm{~s}, 6 \mathrm{H}, \mathrm{OCH}_{3}\right) ; 6.93-7.96(\mathrm{~m}, 8 \mathrm{H}$, Aromaten), ${ }^{13} \mathrm{C}$-NMR ( $\mathrm{CDCl}_{3}$, Raumtemp.): $\delta=273.4$ $\left(\mathrm{C}_{\alpha}\right) ; 195.3$ (cis-CO; trans-CO: n. gef.); $157.8\left(\mathrm{C}_{\gamma}\right)$; $137.8\left(\mathrm{C}_{\beta}\right) ; 163.4 ; 134.0 ; 130.5 ; 114.8$ (Aromaten); 55.4 $\left(\mathrm{OCH}_{3}\right)$. UV-VIS $\left(\mathrm{CH}_{2} \mathrm{Cl}_{2}\right.$, Raumtemp.): $\lambda_{\text {max }}(\log \epsilon)$ $=426 \mathrm{~nm}$ (4.07), 660 (4.08). Aufgrund der Zersetzlichkeit von 1c bei Raumtemp. konnte bislang keine befriedigende Elementaranalyse erhalten werden. [Bis(p-dimethylaminophenyl)allenyliden] (pentacarbonyl) wolfram (1d). Chromatographie zuerst mit $\mathrm{Et}_{2} \mathrm{O}$, dann mit $\mathrm{Et}_{2} \mathrm{O} / \mathrm{THF}$-Gemischen und schliesslich mit reinem THF an Kieselgel bei $-20^{\circ} \mathrm{C}$. 1d wird nach einem braunen Vorlauf als intensiv blaue Zone eluiert und aus $30 \mathrm{ml}$ Pentan $/ \mathrm{CH}_{2} \mathrm{Cl}_{2}(1 / 5)$ kristallisiert. Goldglänzendes, schwarzes Pulver. Ausb. $1.38 \mathrm{~g} \mathrm{(46 \%}$ bez. auf W(CO) $\left.)_{6}\right)$. Zers. ab $150^{\circ} \mathrm{C}$. IR $\left(\mathrm{CH}_{2} \mathrm{Cl}_{2}\right): \nu(\mathrm{CO})$ $2070 \mathrm{w}, 1959 \mathrm{~s}, 1930 \mathrm{vs} \mathrm{cm} \mathrm{cm}^{-1} ; \nu$ (CCC) $1907 \mathrm{ssh} \mathrm{cm}^{-1}$. ${ }^{1} \mathrm{H}-\mathrm{NMR}\left(\mathrm{CDCl}_{3}\right.$, Raumtemp.): $\delta=3.14 \quad(\mathrm{~s}, 12 \mathrm{H}$, $\left.\mathrm{N}\left(\mathrm{CH}_{3}\right)_{2}\right) ; 6.67-7.93\left(\mathrm{~m}, 8 \mathrm{H}\right.$, Aromaten). ${ }^{13} \mathrm{C}-\mathrm{NMR}$ $\left(\mathrm{CD}_{2} \mathrm{Cl}_{2}\right.$, Raumtemp.): $\delta=259.2\left(\mathrm{C}_{\alpha}\right) ; 197.7$ (cis-CO, $J$ (WC) $102 \mathrm{~Hz}$; trans-CO: n. gef.); $161.0\left(\mathrm{C}_{\gamma}\right) ; 147.9$ $\left(\mathrm{C}_{\beta}\right) ; 154.7 ; 136.9 ; 131.1 ; 112.2$ (Aromaten); 40.6 $\left(\mathrm{N}\left(\mathrm{CH}_{3}\right)_{2}\right)$. UV-VIS $\left(\mathrm{CH}_{2} \mathrm{Cl}_{2}\right.$, Raumtemp.): $\lambda_{\max }$ $(\log \epsilon)=402 \mathrm{~nm}$ (3.85), 572 (4.86), 658 (4.77). Analyse: Gef.: C, 48.00; H, 3.43; N, 4.67; Mol.-Gew. 600 (EI-MS, $\left.{ }^{184} \mathrm{~W}\right) . \mathrm{C}_{24} \mathrm{H}_{20} \mathrm{~N}_{2} \mathrm{O}_{5} \mathrm{~W}$ ber.: C, 48.02; H, 3.36; N, 4.67\%; Mol.-Gew. 600.3.

\subsubsection{Darstellung von $1 d$ und $2 c-e$}

Eine aus $5 \mathrm{mmol} \mathrm{M}(\mathrm{CO})_{6}$ dargestellte Lösung von $(\mathrm{CO})_{5} \mathrm{M}[\mathrm{THF}]$ in $\mathrm{THF}$ wird bei Raumtemp. mit 5 mmol des entsprechenden Propargylalkoholes (1d: 1.47 g 1,1-Bis ( $p$-dimethylaminophenyl)prop-2-in-1-ol, 2c: $1.14 \mathrm{~g}$ 9-Ethinyl-9-hydroxyxanthen, 2d: $1.34 \mathrm{~g}$ 1,1Bis( $p$-methoxyphenyl)prop-2-in-1-ol, 2e: $1.47 \mathrm{~g}$ 1,1Bis( $p$-dimethylaminophenyl)prop-2-in-1-ol)) versetzt und $1 \mathrm{~h}$ gerührt. Die blaue Lösung wird im Vakuum 
zur Trockne gebracht und der Rückstand in $50 \mathrm{ml}$ $\mathrm{CH}_{2} \mathrm{Cl}_{2}$ aufgenommen. Nach einer Stunde Rühren bei Raumtemp. wird das Lösungsmittel im Vakuum cntfernt und der Rückstand bei $-20^{\circ} \mathrm{C}$ an Kieselgel chromatographiert.

/Bis(p-dimethylaminophenyl)allenyliden/(pentacarbonyl)wolfram (Id). Chromatographie zuerst mit $\mathrm{Et}_{2} \mathrm{O}$, dann mit $\mathrm{Et}_{2} \mathrm{O} / \mathrm{THF}-\mathrm{Gemischen}$ und schliesslich mit reinem THF. Die blauschwarze Zone enthält Id. Nach Umkristallisieren aus $20 \mathrm{ml}$ Pentan $/ \mathrm{CH}_{2} \mathrm{Cl}_{2}(1 / 4)$ erhält man ein golden glänzendes, schwarzes Pulver. Ausb. $2.1 \mathrm{~g}\left(70 \%\right.$ bez. auf $\mathrm{W}(\mathrm{CO})$ ). Zers. ab $150^{\circ} \mathrm{C}$. Spektroskopische Daten siche bei 5.2.1.: Id).

/Bis(p-methoxyphenyl)allenyliden/(pentacarbonyl)chrom (2c). Nach Chromatographic mit Et, $\mathrm{O}$ und Kristallisation aus $5 \mathrm{ml}$ Pentan $/ \mathrm{CH}_{2} \mathrm{Cl}_{2}(2 / 1)$ wird $2 \mathrm{c}$ in Form von schwarzen Kristallen erhalten. Ausb. $0.22 \mathrm{~g}(26 \%$ bez. auf $\left.\mathrm{Cr}(\mathrm{CO})_{0}\right)$. Zers. ab $150^{\circ} \mathrm{C}$. IR $(\mathrm{Et}, \mathrm{O}): v(\mathrm{CO})$ $2063 \mathrm{~m}, 1971 \mathrm{~m}, 1960 \mathrm{~m}, 1934 \mathrm{vs} \mathrm{cm}^{-1}{ }^{-1}{ }^{1} \mathrm{H}-\mathrm{NMR}\left(\mathrm{CDCl}_{3}\right.$, Raumtemp.): $\delta=3.91$ (s, 6H, OCH $)_{3} ; 6.95-7.86(\mathrm{~m}$. $8 \mathrm{H}$, Aromaten). ${ }^{13} \mathrm{C}-\mathrm{NMR}$ (CDCl${ }_{3}$, Raumtemp.): $\delta=$ $313.9\left(\mathrm{C}_{c}\right) ; 235.3$ (trans-CO); 215.8 (cis-CO); 163.4 $\left(C_{\gamma}\right) ; 135.5\left(C_{\beta}\right) ; 157.0 ; 134.5 ; 114.2$ (Aromaten); 55.7 $\left(\mathrm{OCH}_{3}\right)$. UV-VIS $\left(\mathrm{CH}_{2} \mathrm{Cl}_{2}\right.$, Raumtemp.): $\lambda_{\max }(\log \epsilon)$ $=680 \mathrm{~nm}(5.33), 422(5.21)$. Analyse: Gef: $\mathrm{C}, 59.32 ; \mathrm{H}$, 3.32; Mol.-Gew. 442 (El-MS). $\mathrm{C}_{2} \mathrm{H}_{14} \mathrm{CrO}_{2}$ ber: $\mathrm{C}$, $59.73 ; \mathrm{H}, 3.19 \%$; Mol.-Gew. 442.3.

/Bis(p-dimethylaminophenyl)allenyliden/(pentacarbo$n y$ l)chrom (2d). Es wird solange mit Et, O eluiert, bis alle braun gefärbten Verunreinigungen abgetrennt sind. Danach wird mit THF chromatographiert. Nach Einengen der tiefblauen Produktfraktion zur Trockne verbleibt $\mathbf{2 d}$ in Form eines schwarzen Pulvers. Ausb. $1.80 \mathrm{~g}\left(77 \%\right.$ bez. auf $\left.\mathrm{Cr}(\mathrm{CO})_{6}\right)$. Zers. ab $148^{\circ} \mathrm{C}$. IR $\left(\mathrm{Et}_{2} \mathrm{O}\right): v(\mathrm{CO}) 206.3 \mathrm{w}, 1961 \mathrm{~m}, 1944 \mathrm{vs}, 1920 \mathrm{vs} \mathrm{cm}^{-1}$. ${ }^{1} \mathrm{H}-\mathrm{NMR}\left(\mathrm{CDC}_{3}, 0^{\circ} \mathrm{C}\right): \delta=3.24\left(\mathrm{~s}, 12 \mathrm{H} . \mathrm{N}\left(\mathrm{CH}_{3}\right)_{2}\right)$; $6.89-7.87\left(\mathrm{~m}, 8 \mathrm{H}\right.$, Aromaten). ${ }^{13} \mathrm{C}-\mathrm{NMR}\left(\mathrm{CDCl}_{3}, 0^{\circ} \mathrm{C}\right)$ : $\delta=261.0\left(\mathrm{C}_{\alpha}\right) ; 229.8($ trans-CO$) ; 217.4(\mathrm{cts}-\mathrm{CO}) ; 153.5$ $\left(\mathrm{C}_{\gamma}\right) ; 136.3\left(\mathrm{C}_{\beta}\right) ; 155.2 ; 130.2 ; 126.9 ; 111.3$ (Aromaten); $40.3\left(\mathrm{~N}\left(\mathrm{CH}_{3}\right)_{2}\right)$. UV-VIS $\left(\mathrm{CH}_{2} \mathrm{Cl}_{2}\right.$. Raumtemp. $): \lambda_{\text {max }}$ $(\log \epsilon)=686 \mathrm{~nm}(4.56), 560(4.73), 410(3.87)$. Analyse: Gef: C, 61.72; H, 4.28; N. 5.85; $\mathrm{C}_{24} \mathrm{H}_{20} \mathrm{CrN}_{2} \mathrm{O}_{5}$ ber.: C, 61.54; H, 4.30; N. 5.98\%; Mol.-Gew. 468.4.

Pentacarbony//3,3-o,o'-diphenyletherdiylallenyliden/-

chrom (2e). Nach Chromatographie mit $\mathrm{Et}_{2} \mathrm{O}$ und Umkristallisieren aus $25 \mathrm{ml} \mathrm{CH}_{2} \mathrm{Cl}_{2}$ erhält man $2 \mathrm{e}$ in Form von goldglänzenden, schwarzen Nadeln. Ausb. $1.38 \mathrm{~g}\left(70 \%\right.$ bez. auf $\mathrm{Cr}(\mathrm{CO})$ ). Schmp. $145^{\circ} \mathrm{C}$. IR $\left(\mathrm{CH}_{2} \mathrm{Cl}_{2}\right): \nu(\mathrm{CO}) 2063 \mathrm{~m}, 1958 \mathrm{msh}, 1938 \mathrm{vs} \mathrm{cm}{ }^{1}$. ${ }^{1} \mathrm{H}-$ NMR $\left(\mathrm{CDCl}_{3}, 0^{\circ} \mathrm{C}\right): \delta=7.45-8.33(\mathrm{~m}) .{ }^{10} \mathrm{C}-\mathrm{NMR}$ $\left(\mathrm{CDCl}_{3}, 10^{\circ} \mathrm{C}\right): \delta=290.6\left(\mathrm{C}_{i t}\right) ; 232.6($ trans-CO$): 216.2$ $\left(\right.$ cis-CO); $169.3\left(\mathrm{C}_{\gamma}\right) ; 135.1\left(\mathrm{C}_{\beta}\right) ; 154.2 ; 139.2 ; 129.7$; $126.2 ; 125.8 ; 118.3$ (Aromaten). UV-VIS $\left(\mathrm{CH}_{2} \mathrm{Cl}_{2}\right.$, Raumtemp.): $\lambda_{\max }(\log \epsilon)=732 \mathrm{~nm}$ (4.51). Analyse:
Gef:: C, 60.76; H. 2.06; Mol-Gew. 396 (FAB-MS, $\mathrm{NBOH}) . \mathrm{C}_{3 i} \mathrm{H}_{8} \mathrm{CrO}_{6}$ her.: $\mathrm{C}, 60.62 ; \mathrm{H}, 2.03 \%$; Mol. Gew. 396.3.

\subsection{Reaktionen ion la-d und 2d mit Diethylamin}

\subsubsection{Umsetzungen ron $1 a-c$}

$\mathrm{Zu}$ den aus $5 \mathrm{mmol} \mathrm{W}(\mathrm{CO})_{6}$ nach 5.2.1. dargestellten Lösungen von $\mathbf{1 a}, \mathbf{l b}$ und $\mathbf{1 c}$ in $25 \mathrm{ml} \mathrm{CH}_{2} \mathrm{Cl}_{2}$ werden bei $-70^{\circ} \mathrm{C}$ unter Rühren $0.53 \mathrm{ml}(5 \mathrm{mmol})$ HNEt, zugesetzt. Bei der Zugabe schlägt die Farbe nach braun um. Dats jeweilige Reaktionsgemisch wird auf Raumtemp. erwärmt. im Vakuum zur Trockne gebracht und der Rückstand an Kieselgel bei $-20^{\circ} \mathrm{C}$ chromatographicrt.

Pentacarbony//1-diethylamino-3,3-diphenyl)prop-2-enyliden/wolfram (3a). 3a wird zucrst mit Pentan, danach mit Pentan/ $\mathrm{CH}, \mathrm{Cl}$, (autsteigend polar bis 8/2) cluiert. Nach wenig violettem Vorlaut kann 3a als grünlich-gelbe Zone abgetrennt und im Vakuum zur Trockne gebracht werden. Umkristallisation aus $30 \mathrm{ml}$ Pentan $/ \mathrm{CH}, \mathrm{Cl},(10 / 1)$ liefert $3 \mathrm{a}$ in Form von gelben Kristallen. Ausb. $0.97 \mathrm{~g}(339 \mathrm{bez}$ auf W(CO) $)$. Schmp. 110-111 C. IR (n-Pentan): ${ }^{\circ}(\mathrm{CO}) 2061 \mathrm{w}, 1967 \mathrm{vw}$, 1934vs, 1928vs cm ' ' H-NMR (CDCl, Raumtemp.): $\delta=1.15(6.7 .2 \mathrm{~Hz}, 3 \mathrm{H}, \mathrm{CH},-\mathrm{CH},) ; 1.28(1,7.2 \mathrm{~Hz}, 3 \mathrm{H}$, $\mathrm{CH},-\mathrm{CH}, 3.29\left(\mathrm{dq}, 13.3,7.2 \mathrm{~Hz}, 1 \mathrm{H}, \mathrm{CH}_{2}\right) ; 3.99$ $\left(\mathrm{m}, 2 \mathrm{H}, \mathrm{CH}_{2}\right): 4.38\left(\mathrm{dq}, 13.3,7.2 \mathrm{~Hz}, 1 \mathrm{H}, \mathrm{CH}_{2}\right): 6.82(\mathrm{~s}$, $1 \mathrm{H},=\mathrm{CH}) ; 7.10-7.45(\mathrm{~m}, 10 \mathrm{H}$, Aromaten $){ }^{13} \mathrm{C}-\mathrm{NMR}$ $\left(\mathrm{CDCl}_{3}\right.$, Raumtemp): $\delta=249.8$ (Carben-C): 203,0 $($ trans-CO $): 198.2 \quad($ cis-CO $): 137.6 \quad(=\mathrm{CH}) ; \quad 129.7$

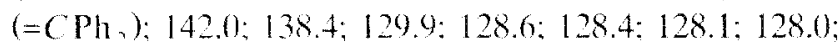
127.7 (Aromaten); $56.7(\mathrm{CH},) ; 7.5\left(\mathrm{CH}_{2}\right) ; 13.2\left(\mathrm{CH}_{3}\right)$ $12.9\left(\mathrm{CH}_{3}\right)$. Analyse: Gef: $\mathrm{C}, 49.82: \mathrm{H}_{4}, 4.02: \mathrm{N}, 2.46$; Mol-Gew. 587 (EI-MS. ${ }^{44} \mathrm{~W}$ ). $\mathrm{C}, \mathrm{H}_{2} \mathrm{NO}_{5} \mathrm{~W}$. 1/6 $\mathrm{C}_{6} \mathrm{H}_{12}$ ber.: $\mathrm{C}, 49.76: \mathrm{H}, 3.87: \mathrm{N}, 2.34 \%$; Mol.-Gew. $587.3+12.03$. (3a konnte bislang nicht vollständig lösungsmittelfrei erhalten werden.)

Pentacarbonyl/(1-diethylamino-3,3-bis-p-tolyl)prop-2-enyliden/wolfram $(\mathbf{3 b})$. 3b wird zuerst mit Pentan, danach mit Pentan $/ \mathrm{CH}_{2} \mathrm{Cl}_{2}$ (aufsteigend polar bis $8 / 2$ ) eluiert. Nach wenig violettem Vorlauf kann $\mathbf{3 b}$ als gelbe Zone abgetrennt und zur Trockne gebracht werden. Umkristallisation aus $30 \mathrm{ml}$ Pentan $/ \mathrm{CH}, \mathrm{Cl},(10 / 1)$ liefert $\mathbf{3 b}$ als gelbe Kristalle. Ausb. $1.14 \mathrm{~g}(37 \% \mathrm{bcz}$ a a f W(CO) $)_{0}$. Schmp. $106-108^{\circ} \mathrm{C}$ IR (n-Pentan): $\nu(\mathrm{CO})$ $2060 \mathrm{w}, 1967 \mathrm{vw}, 1932 \mathrm{vs} .1928 \mathrm{vs} \mathrm{cm}^{-1}$. ${ }^{1} \mathrm{H}-\mathrm{NMR}\left(\mathrm{CDCl}_{3}\right.$. Raumtemp.): $\delta=1.13\left(\mathrm{t}, 7.3 \mathrm{~Hz}, 3 \mathrm{H} . \mathrm{CH}_{2}-\mathrm{CH}_{3}\right) ; 1.29$ (t, $\left.7.3 \mathrm{~Hz}, 3 \mathrm{H}, \mathrm{CH},-\mathrm{CH}_{3}\right): 2.34$ (s, $3 \mathrm{H}, \mathrm{C}_{6} \mathrm{H}_{4}-\mathrm{CH}$ ); $2.38\left(\mathrm{~s}, 3 \mathrm{H}, \mathrm{C}_{6} \mathrm{H}_{4}-\mathrm{CH},\right) ; 3.27(\mathrm{dq}, 13.4 .7 .3 \mathrm{~Hz}, 1 \mathrm{H}$, $\left.\mathrm{CH}_{2}\right): 3.97\left(\mathrm{~m}, 2 \mathrm{H}, \mathrm{CH}_{2}\right): 4.35(\mathrm{dq}, 13.4,7.3 \mathrm{~Hz}, 1 \mathrm{H}$, $\left(\mathrm{CH}_{2}\right) ; 6.73(\mathrm{~s}, 1 \mathrm{H} .=\mathrm{CH}) ; 6.99-7.25(\mathrm{~m}, 8 \mathrm{H}$, Aromaten $)$. ${ }^{1.3} \mathrm{C}-\mathrm{NMR}(\mathrm{CDCl}$, . Raumtemp) $): \delta=249.9$ (Carben-C): 
203.1 (trans-CO); 198.2 (cis-CO, J(WC) $128 \mathrm{~Hz}$ ); 136.9 $(=\mathrm{CH}) ; 135.6(=C($ Aryl $) 2) ; 139.3 ; 137.9 ; 137.5 ; 129.8$; $129.0 ; 128.7 ; 128.5$ (Aromaten); $56.6\left(\mathrm{CH}_{2}\right) ; 47.4\left(\mathrm{CH}_{2}\right)$; $21.1\left(\mathrm{C}_{6} \mathrm{H}_{4}-\mathrm{CH}_{3}\right) ; 13.2\left(\mathrm{CH}_{2}-\mathrm{CH}_{3}\right) ; 12.9\left(\mathrm{CH}_{2}-\mathrm{CH}_{3}\right)$. Analyse: Gef.: C, 50.79; H, 4.12; N, 2.26; Mol.-Gew. $615\left(\right.$ EI-MS, $\left.{ }^{184} \mathrm{~W}\right) . \mathrm{C}_{26} \mathrm{H}_{25} \mathrm{NO}_{5} \mathrm{~W}$ ber.: $\mathrm{C}, 50.75 ; \mathrm{H}$, 4.10; N, 2.28\%; Mol.-Gew. 615.3.

Pentacarbonyl/(1-diethylamino-3,3-bis-p-methoxyphenyl)prop-2-enyliden/wolfram (3c). $3 \mathrm{c}$ wird zuerst mit Pentan, danach mit Pentan $/ \mathrm{CH}_{2} \mathrm{Cl}_{2}$ (aufsteigend polar bis $3 / 7)$ eluiert. Nach wenig gelblichem Vorlauf kann 3c als intensiv gelbe Zone abgetrennt und zur Trockne gebracht werden. Umkristallisation aus $30 \mathrm{ml}$ Pentan $/ \mathrm{CH}_{2} \mathrm{Cl}_{2}(3 / 2)$ liefert $3 \mathrm{c}$ als gelbe Kristalle. Ausb. $1.58 \mathrm{~g}\left(49 \%\right.$ bez. auf $\left.\mathrm{W}(\mathrm{CO})_{6}\right)$. Schmp. 101$102^{\circ} \mathrm{C}$. IR (n-Pentan): $\nu(\mathrm{CO})=2061 \mathrm{w}, 1967 \mathrm{vw}, 1933 \mathrm{vs}$, 1926vs cm ${ }^{-1} .{ }^{1} \mathrm{H}-\mathrm{NMR}\left(\mathrm{CDCl}_{3}\right.$, Raumtemp.): $\delta=1.14$ (t, $\left.7.3 \mathrm{~Hz}, 3 \mathrm{H}, \mathrm{CH}_{2}-\mathrm{CH}_{3}\right) ; 1.31\left(\mathrm{t}, 7.3 \mathrm{~Hz}, 3 \mathrm{H}, \mathrm{CH}_{2}-\right.$ $\left.\mathrm{CH}_{3}\right) ; 3.24\left(\mathrm{dq}, 13.4,7.3 \mathrm{~Hz}, 1 \mathrm{H}, \mathrm{CH}_{2}\right) ; 3.80(\mathrm{~s}, 3 \mathrm{H}$, $\left.\mathrm{OCH}_{3}\right) ; 3.83\left(\mathrm{~s}, 3 \mathrm{H}, \mathrm{OCH}_{3}\right) ; 3.97\left(\mathrm{~m}, 2 \mathrm{H}, \mathrm{CH}_{2}\right) ; 4.35$ $\left(\mathrm{dq}, 13.4,7.3 \mathrm{~Hz}, 1 \mathrm{H}, \mathrm{CH}_{2}\right) ; 6.67(\mathrm{~s}, 1 \mathrm{H},=\mathrm{CH}) ; 6.79-$ $7.25\left(\mathrm{~m}, 8 \mathrm{H}\right.$, Aromaten). ${ }^{13} \mathrm{C}-\mathrm{NMR}\left(\mathrm{CDCl}_{3}\right.$, Raumtemp.): $\delta=249.8$ (Carben-C); 203.1 (trans-CO); 198.2 (cis-CO, J(WC) $128 \mathrm{~Hz}) ; 136.1(=\mathrm{CH}) ; 134.7$ $\left(=C(\text { Aryl })_{2}\right) ; 159.4 ; 159.3 ; 131.2 ; 131.1 ; 129.8 ; 129.5$; $113.7 ; 113.5$ (Aromaten); $56.6\left(\mathrm{CH}_{2}\right) ; 55.3\left(\mathrm{OCH}_{3}\right)$; $47.3\left(\mathrm{CH}_{2}\right) ; 13.2\left(\mathrm{CH}_{2}-\mathrm{CH}_{3}\right) ; 13.0\left(\mathrm{CH}_{2}-\mathrm{CH}_{3}\right)$. Analyse: Gef.: $\mathrm{C}, 48.14 ; \mathrm{H}, 3.88 ; \mathrm{N}, 2.09 . \mathrm{C}_{26} \mathrm{H}_{25} \mathrm{NO}_{7} \mathrm{~W}$ ber.: C, 48.24; H, 3.89; N, 2.16\%; Mol.-Gew. 647.4.

\subsubsection{Reaktionen von $1 d$ und $2 d$}

Pentacarbonyl/(1-diethylamino-3,3-bis-p-dimethylaminophenyl)prop-2-enyliden/wolfram (3d). $180 \mathrm{mg}(0.3$ mmol) 1d werden in $20 \mathrm{ml} \mathrm{CH} \mathrm{Cl}_{2}$ gelöst und bei Raumtemp. mit $0.03 \mathrm{ml}(0.3 \mathrm{mmol}) \mathrm{HNEt}_{2}$ versetzt. $2 \mathrm{~s}$ nach der Zugabe ist das Reaktionsgemisch braun. Die Lösung wird zur Trockne gebracht und der Rückstand an Kieselgel bei $-20^{\circ} \mathrm{C}$ chromatographiert. Es wird zuerst mit Pentan, danach mit Et ${ }_{2} \mathrm{O} /$ Pentan $/ \mathrm{CH}_{2} \mathrm{Cl}_{2}$ (aufsteigend polar bis $1 / 3 / 6$ ) eluiert. 3d kann als gelbe Zone abgetrennt und zur Trockne gebracht werden. Umkristallisation aus $15 \mathrm{ml}$ Pentan $/ \mathrm{CH}_{2} \mathrm{Cl}_{2}(2 / 1)$ liefert 3d in Form eines gelben Pulvers. Ausb. $90 \mathrm{mg}$ ( $45 \%$ bez. auf $\mathrm{W}(\mathrm{CO})_{6}$ ). Zers. ab $120^{\circ} \mathrm{C}$. IR (n-Pentan): $\nu$ (CO) 2060w, 1966w, 1930m, 1922vs cm ${ }^{-1}$. ${ }^{1} \mathrm{H}-\mathrm{NMR}$ $\left(\mathrm{CDCl}_{3}\right.$, Raumtemp.): $\delta=1.12\left(\mathrm{t}, 7.2 \mathrm{~Hz}, 3 \mathrm{H}, \mathrm{CH}_{2}-\right.$ $\left.\mathrm{CH}_{3}\right) ; 1.31\left(\mathrm{t}, 7.2 \mathrm{~Hz}, 3 \mathrm{H}, \mathrm{CH}_{2}-\mathrm{CH}_{3}\right) ; 2.95(\mathrm{~s}, 6 \mathrm{H}$, $\left.\mathrm{N}\left(\mathrm{CH}_{3}\right)_{2}\right) ; 2.99\left(\mathrm{~s}, 6 \mathrm{H}, \mathrm{N}\left(\mathrm{CH}_{3}\right)_{2}\right) ; 3.21(\mathrm{dq}, 13.5,7.1$ $\left.\mathrm{Hz}, 1 \mathrm{H}, \mathrm{CH}_{2}\right) ; 3.96\left(\mathrm{~m}, 2 \mathrm{H}, \mathrm{CH}_{2}\right) ; 4.35(\mathrm{dq}, 13.3,7.1$ $\left.\mathrm{Hz}, 1 \mathrm{H}, \mathrm{CH}_{2}\right) ; 6.58(\mathrm{~s}, 1 \mathrm{H},=\mathrm{CH}) ; 6.58-7.26(\mathrm{~m}, 8 \mathrm{H}$, Aromaten $).{ }^{13} \mathrm{C}$-NMR $\left(\mathrm{CDCl}_{3},-10^{\circ} \mathrm{C}\right): \delta=249.1$ (Carben-C); 203.7 (trans-CO); 198.3 (cis-CO), J(WC) $128 \mathrm{~Hz}) ; 134.2$ (=CH); 149.8; 131.1; 130.3; 129.7; 126.6;
111.7; 111.3 [Aromaten $+=C(\text { Aryl })_{2}$ ]; $56.5\left(\mathrm{CH}_{2}\right) ; 46.8$ $\left(\mathrm{CH}_{2}\right) ; 40.5\left(\mathrm{~N}\left(\mathrm{CH}_{3}\right)_{2}\right) ; 40.4\left(\mathrm{~N}\left(\mathrm{CH}_{3}\right)_{2}\right) ; 13.3\left(\mathrm{CH}_{2}-\right.$ $\left.\mathrm{CH}_{3}\right)$; $13.1\left(\mathrm{CH}_{2}-\mathrm{CH}_{3}\right)$. Analyse: Gef.: $\mathrm{C}, 49.67 ; \mathrm{H}$, 4.63; $\mathrm{N}, 6.08 . \mathrm{C}_{28} \mathrm{H}_{31} \mathrm{~N}_{3} \mathrm{O}_{5} \mathrm{~W}$ ber.: $\mathrm{C}, 49.94 ; \mathrm{H}, 4.64 ; \mathrm{N}$, 6.24\%; Mol.-Gew. 673.4.

Pentacarbonyl/(1-diethylamino-3,3-bis-p-dimethylaminophenyl)prop-2-enyliden/chrom (4d). $\mathrm{Zu}$ einer Lösung von $0.50 \mathrm{~g}(1.1 \mathrm{mmol}) 2 \mathrm{~d}$ in $20 \mathrm{ml} \mathrm{CH}{ }_{2} \mathrm{Cl}_{2}$ tropft man bei Raumtemp. bis zur Gelbfärbung $(0.1 \mathrm{ml}$ (1.4 mmol)) Diethylamin. Nach chromatographischer Aufarbeitung des Reaktionsgemisches an Kieselgel bei $-20^{\circ} \mathrm{C}$ mit Pentan $/ \mathrm{Et}_{2} \mathrm{O}(1 / 1)$ lässt sich $4 \mathbf{d}$ als gelbes Pulver isolieren. Ausb. $0.52 \mathrm{~g}(88 \%$ bez. auf $2 \mathrm{~d})$. Schmp. $140^{\circ} \mathrm{C}$ (Zers.). IR (n-Pentan): $\nu$ (CO) $2050 \mathrm{w}, 1968 \mathrm{w}$, 1926vs, $1910 \mathrm{msh} \mathrm{cm}^{-1} .{ }^{1} \mathrm{H}-\mathrm{NMR}\left(\mathrm{CDCl}_{3},-10^{\circ} \mathrm{C}\right): \delta$ $=1.08\left(\mathrm{t}, 7.1 \mathrm{~Hz}, 3 \mathrm{H}, \mathrm{CH}_{2}-\mathrm{CH}_{3}\right) ; 1.30(\mathrm{t}, 7.2 \mathrm{~Hz}, 3 \mathrm{H}$, $\left.\mathrm{CH}_{2}-\mathrm{CH}_{3}\right) ; 2.93\left(\mathrm{~s}, 6 \mathrm{H}, \mathrm{N}\left(\mathrm{CH}_{3}\right)_{2}\right) ; 2.94\left(\mathrm{~m}, 1 \mathrm{H}, \mathrm{CH}_{2}\right)$; $2.96\left(\mathrm{~s}, 6 \mathrm{H}, \mathrm{N}\left(\mathrm{CH}_{3}\right)_{2}\right) ; 3.13\left(\mathrm{~m}, 1 \mathrm{H}, \mathrm{CH}_{2}\right) ; 3.99(\mathrm{~m}, 1 \mathrm{H}$, $\left.\mathrm{CH}_{2}\right) ; 4.40\left(\mathrm{~m}, 1 \mathrm{H}, \mathrm{CH}_{2}\right) ; 6.67(\mathrm{~s}, 1 \mathrm{H},=\mathrm{CH}) ; 6.56-7.24$ (m, 8H, Aromaten). ${ }^{13} \mathrm{C}$-NMR $\left(\mathrm{CDCl}_{3},-10^{\circ} \mathrm{C}\right): \delta=$ 267.1 (Carben-C); 223.8 (trans-CO); 217.3 (cis-CO); $149.8 ; 149.7 ; 130.8 ; 129.6 ; 128.9 ; 126.9 ; 111.8 ; 111.3$ (Aromaten); $133.5 \quad(=\mathrm{CH}) ; 130.6 \quad\left[=C(\text { Aryl })_{2}\right] ; 54.5$ $\left(\mathrm{CH}_{2}\right) ; 48.0\left(\mathrm{CH}_{2}\right) ; 13.4\left(\mathrm{CH}_{2}-\mathrm{CH}_{3}\right) ; 13.3\left(\mathrm{CH}_{2}-\mathrm{CH}_{3}\right)$. Analyse: Gef.: $\mathrm{C}, \quad 62.02 ; \mathrm{H}, \quad 5.84 ; \mathrm{N}, 7.69$. $\mathrm{C}_{28} \mathrm{H}_{31} \mathrm{CrN}_{3} \mathrm{O}_{5}$ ber.: $\mathrm{C}, 62.10 ; \mathrm{H}, 5.77$; N, 7.76\%; Mol.Gew. 541.5.

\subsection{Reaktionen von 1 a und 1 d mit Anilin}

Pentacarbonyl/(1-phenylamino-3,3-diphenyl)prop-2-enyliden/wolfram (5a). Eine Lösung von nach 5.2 .1 aus 5 mmol W(CO) $)_{6}$ dargestelltem 1a in $20 \mathrm{ml} \mathrm{CH}_{2} \mathrm{Cl}_{2}$ wird bei $-20^{\circ} \mathrm{C}$ mit $0.46 \mathrm{ml}(5 \mathrm{mmol})$ Anilin versetzt und unter Rühren innerhalb einer Stunde auf Raumtemp. erwärmt. Die braune Lösung wird danach im Vakuum zur Trockne eingeengt und an Kieselgel bei $-20^{\circ} \mathrm{C}$ chromatographiert. 5a wird zuerst mit Pentan, danach mit Pentan $/ \mathrm{CH}_{2} \mathrm{Cl}_{2}$ (aufsteigend polar bis 7/3) eluiert. Nach einem violetten und einem gelben Vorlauf, der im IR-Spektrum keine CO-Schwingungen zeigt, kann 5a als orange Zone abgetrennt und zur Trockne gebracht werden. Umkristallisation aus $15 \mathrm{ml}$ Pentan/ $\mathrm{CH}_{2} \mathrm{Cl}_{2}(9 / 1)$ liefert 5a in Form von orangen Kristallen. Ausb. $270 \mathrm{mg}(9 \% \text { bez. auf W(CO) })_{6}$. Schmp. 103$105^{\circ} \mathrm{C}$. IR (n-Pentan): $\nu(\mathrm{CO}) 2062 \mathrm{w}, 1973 \mathrm{vw}, 1942 \mathrm{vs}$, 1933vs $\mathrm{cm}^{-1}$. ${ }^{1} \mathrm{H}-\mathrm{NMR}\left(\mathrm{CDCl}_{3}\right.$, Raumtemp.): $\delta=$ 6.75-7.33 (m, 16H, Ph $+=\mathrm{CH}$ ); 9.88 (s br., $1 \mathrm{H}, \mathrm{NH}$ ). ${ }^{13} \mathrm{C}-\mathrm{NMR}\left(\mathrm{CDCl}_{3}\right.$, Raumtemp.): $\delta=258.8$ (Carben-C); 203.1 (trans-CO); 198.2 (cis-CO, $J(\mathrm{WC}) 127 \mathrm{~Hz}$ ); 141.5; $140.1 ; 138.3(\mathrm{Ph}) ; 136.3(=\mathrm{CH}) ; 135.0\left(=C \mathrm{Ph}_{2}\right) ; 129.7$; $129.1 ; 128.5 ; 128.4 ; 128.2 ; 128.1 ; 127.6 ; 122.9(\mathrm{Ph})$. Analyse: Gef.: $\mathrm{C}, 51.21 ; \mathrm{H}, 2.86 ; \mathrm{N}, 2.36 . \mathrm{C}_{26} \mathrm{H}_{17} \mathrm{NO}_{5} \mathrm{~W}$ ber.: C, 51.42; H, 2.82; N, 2.31\%; Mol.-Gew. 607.3. E-/Z-Pentacarbonyl/(1-phenylamino-3,3-bis-p-dimethyl- 
aminophenyl)prop-2-enyliden/wolfram (5d). Enc lösung von $180 \mathrm{mg}(0.30 \mathrm{mmol})$ 1d in $15 \mathrm{mlCH}, \mathrm{Cl}$, wird bei Raumtemp. mit $0.30 \mathrm{ml}(0.33 \mathrm{mmol})$ Anitin versetzt und 48 h gerühr. Nach Entfernen des Losungsmittels wird der Rückstand bei Raumtemp. an Kieselgel chromatographient. Mit Pentan/Et $01 / 1)$ wird $5 d$ als orange Zone cluiert. Durch Umkristallisatom ans 10 ml Pentan $\mathrm{CH}, \mathrm{Cl},(8 / 2)$ erhalt man $5 \mathrm{~d}$ in form von orangen kristallen. 5d stell ein $\ell-/ z$-lsomerenge misch dar, das bislang chromatographisch nicht autgetrennt werden konnte. Ausb. $50 \mathrm{mg}(23 \%$, beg. auf id) Schmp. 121-122( (Zers). IR (Lt,O) , (6) 1433ks. 1915msh. 2059w. 1960vw cm! H-NMR CODCl, Raumtemp.): $\quad s=2.97 ; 2.99: 3.11$ \& s. $12 \mathrm{H}$, $\left.\mathrm{N}\left(\mathrm{CH}_{3}\right)_{2}\right) ; 6.53-7.38(\mathrm{~m} .14 \mathrm{H}$. Aromaten $=(\mathrm{H}) ; 9.66$ (s br. 1H. NH), (C-NMR (CDCl, Ramtemp): 8 254.4 (Carben-C): 204. l trans-CO): 198.4 is-CO. $J(W C) 127 \mathrm{~Hz}) ; 150.6: 150.3 ; 142.7 ; 140.8: 140.6 ; 1392$ : $137.8 ; 132.6 ; 131.8 ; 131.7 ; 130.2 ; 130.0 ; 129.7 ; 129.2 \%$ $128.9 ; 128.7 ; 126.6: 125.6 ; 1228: 112.1: 111.7: 111.6$ [Aromaten $+=\mathrm{CH}+=\mathrm{C}(\mathrm{Aryl})$, ] $40.4(\mathrm{~N}(\mathrm{CH}), 4), 40.3$ $\left(\mathrm{N}\left(\mathrm{CH}_{3}\right), 40.2\left(\mathrm{~N}\left(\mathrm{CH}_{3}, 3\right)\right.\right.$. Analyse: Gef: $\mathrm{C}, 49.96 ; \mathrm{H}$, $3.71: \mathrm{N}, 5.91 . \mathrm{C}_{3} \mathrm{H}_{27} \mathrm{~N}_{3} \mathrm{O}, \mathrm{W} \cdot 1 / 2 \mathrm{CH} \cdot \mathrm{Cl} \mathrm{ber}_{2} \mathrm{C}$, 49.78; H. 3.84; N. 5.71\%; Mol.-Gow. 693.4+42.4. $5 d$ konnte bislang nicht vollständig lösungsmintelfei erhalten werden.)

\subsection{Reaktionen ion la. Ib and Id mit Benzophenonimin und lon $2 \mathrm{e}$ mit $H N=C(B u)-p-T o l y l$}

Die Lösungen von nach 5.2. . aus 5 mmol W(CO) dargestellem $\mathbf{l a}, \mathbf{l b}$ und $\mathbf{I d}$ in $50 \mathrm{ml} \mathrm{CH}, \mathrm{Cl}$, werden bei -25 C (1a und 1b) bzw. bei Raumtemp. (1d) mit jeweils $0.84 \mathrm{ml}(5 \mathrm{mmol})$ Benzophenonimin versetzt. Die Reaktionsgemische werden unter Ruhren innerhalb von 2 h auf Ratumtemp. crwämt ( $\mathbf{l}$ a und $\mathbf{l b}$ ) bzw. bei Raumtemp. 12 h gerührt (1d). Dic braunen Lösungen werden anschliessend zur Trockne gebracht und bei - $20 \mathrm{C}$ an Kieselgel chromatographicn.

Pentacarbonyll 1-diphenymethylidenamino-3,3-diphenyl)prop-2-enyliden/wolfram (6a) and Benzophenonimin pentacarbonyl wolfram. Fis wird zunächst mil Pentan, danach mit Pentan/ $\mathrm{CH}_{2} \mathrm{Cl}$, aufsteigend polar bis $8 / 2$ ) eluiert. Nach cinem geb-brannen Vorlaul, der im IR-Spektrum nur die (O-Schwingung von $\mathrm{W}\left(\mathrm{CO}_{\mathrm{b}}\right.$ zeigt, kann 6a als orange-rote Zone abgetrennt werden. Nachfolgend tritt eine gelbe Fraktion atu, die den Benzophenonimin(pentacarbonyl)wolfram-komplex Liefert (Ausb, $180 \mathrm{mg}(7 \%$ bez. aut W(CO), Schmp. $92-93^{\circ} \mathrm{C}$. Dic spektroskopische Charakterisierung erfolgt durch Vergleich mit den Literaturdaten [29]), 6a wind aus $20 \mathrm{ml}$ Pentan/ $\mathrm{CH}_{2} \mathrm{Cl}_{2}(9 / 1)$ in form von hellroten Kristallen erhalten. Aush. $590 \mathrm{mg} / 17 \%$ bez. auf $\left.\mathrm{W}(\mathrm{CO})_{0}\right)$. Schmp. $157-159^{\circ} \mathrm{C}$. IR (n-Pentan): $v(\mathrm{CO})$ $2060 \mathrm{w}, 1936 \mathrm{sh} .1929 \mathrm{~s}$ in ! $\mathrm{H}-\mathrm{NMR} / \mathrm{CDCl}$
Raumtemp.): $8=6.76-7.55$ (m). $\quad$ C-NMR (CDCl, Raumtemp.): $\quad \delta=203.2$ (trans-CO): 198.5 (cis-CO. f(WC) 128 Hz): 190.1 (carben-C); $44.9(\mathrm{~N}=\mathrm{C}) ; 140.6$ : $137.8: 1.309: 130.0 ; 129.7: 129,1: 1287,128.6 ; 128.5$ 128.3: $128.2 . \quad 127.4$ Aromatent $=$ (H): 124.9 $1=(0$ Aryl) $]$. Analyse: Get. C. 5706; 11. 304: N, 2002:

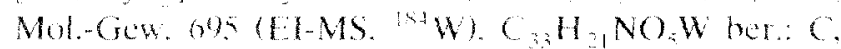
57.00: H. 3014: N. 2017\%: Mol.-Gew. 695.4.

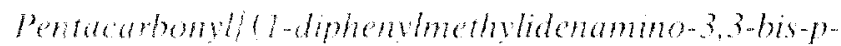
by/prop-2-chylden/wollam (6b). Eswird zunachst mit Pentan, danach mit Pentan $\mathrm{CH}, \mathrm{Cl}$, Aafsteigend poar bis 8 2) aluen. Nach einem violetten Vorlat (laut IR-Spektrum W(CO), kann 6 als orange-rote Zone abgetronnt werden. Nach Entfernen des lösungsmit. tels wird 6 bus $24 \mathrm{~m}$ Pontan/CH Cl, (9/1) in form von helloton kristallon shalten. Ausb. $735 \mathrm{mg}(21 \%$ bez. aut W(CO), Schmp. 116-118\% IR (n-Pentan): 10O) $2059 w, 19345 h$. $1928 \mathrm{sm}$ ! H-NMR (CDCl: Raumtemp. : $8=1.49(\mathrm{~s}, 3 \mathrm{H}, \mathrm{CH}, 2.35(\mathrm{~s}, 3 \mathrm{H}, \mathrm{CH})$ $6.64-7.48$ (m. 101., Aromaten $+=\mathrm{CH}) \quad$ C-NMR (CDCI. Ratumtemp.): $\delta=203+4$ (trans-CO); 198.7 (cis(O). J(WO) $127 \mathrm{~Hz})$ : $189.8(\mathrm{Cahen}-\mathrm{C})$ : $46.5(\mathrm{~N}=\mathrm{C})$;

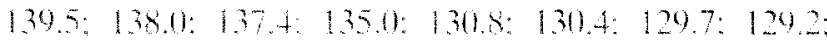
129.1: 128.6: 128.4: 128.2: 127.7 (Aromaten $+=\mathrm{CH}$; 124.6 [ = (Arv) ]: 21,3 (CH, $21.2\left(\mathrm{CH}_{3}\right)$. Analyse: Gef: C.58.23: H, 354: N. 2.20, C $3 H_{2} \mathrm{NO}_{3} \mathrm{~W}$ ber: $\mathrm{C}$. $58.11,11,3.48:$ N. 1.94\%. Mol.-Crew. 723.4.

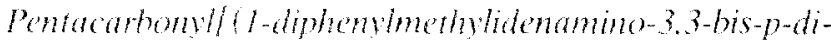

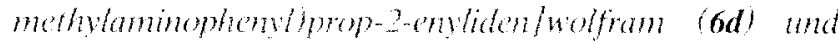
Benaphenomim! pentatahomyllwatham. Es wird zunähst mit Pentan. damach mit Pentan/ $\mathrm{CH}_{2} \mathrm{Cl}_{2}$ (atfsteigend polar his $2 / 1$ ) eluiert. Nach einer gelben Faktion dis don Benzophenonimintpentacarbonyl)wolfran-komplox [2y] (Aust. $105 \mathrm{mg}$ (4\% bez. anf W(CO), Schmp. $92-93(y)$ lictert. kann 6d als rote Zone abgetrenn werden. 6d wird aus $10 \mathrm{ml}$ Pentan $/ \mathrm{CH}, \mathrm{Cl},(7 / 1)$ in fom von schwarzroten Kristallen erhaten. Aush +25 me (11) bez. aut W(CO) ). Schmp. $143-144 \mathrm{C}$. IR $(\mathrm{n}=$ Pentan $): v(\mathrm{CO})$ $2057 \mathrm{w}, 1967 \mathrm{w}, 1932 \mathrm{~m}, 1922 \mathrm{vsm} \mathrm{cm}^{-1}$ 'H-NMR (CDCl Ratumtemp.) o $=2.68(\mathrm{~s} .6 \mathrm{H}, \mathrm{N}(\mathrm{CH} ;), 2.99(\mathrm{~s}, 6 \mathrm{H}$.

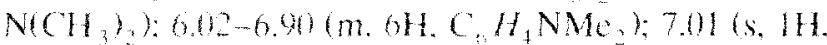
$=\mathrm{CH}) \quad 7.23-744 \mathrm{n}, 12 \mathrm{H}$. Aromaten). $\mathrm{C}-\mathrm{NMR}$ (CDCl, Raumtemp): $\delta=203.9($ trams-CO): $199.2(\mathrm{cs}-$ CO. (WC) LS Hz; 191.4 (Camen-C): $149.5(\mathrm{~N}=\mathrm{C}):$

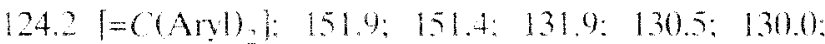
$129.9 ; 129.6 ; 128.7 ; 128.3 ; 125.4 ; 1229 ; 111.6 ; 111.2$ (Aromaten $+\mathrm{CH}): 40.1 \quad\left(\mathrm{~N}\left(\mathrm{CH}_{3}\right)_{2}\right), 39.8\left(\mathrm{~N}\left(\mathrm{CH}_{3}\right)_{2}\right)$. UV-VIS (CH, Cl. Raumtemp $: \lambda_{\text {max }}(\log \epsilon)=420 \mathrm{~nm}$ (4.45). Analyse: Get: ( 56.94: H, 4.05: N. 5.48. $\mathrm{C}_{37} \mathrm{H}_{31} \mathrm{~N}_{3} \mathrm{O}$ W ber: C. 56.86: H. 4.00: N. $5.38 \%:$ Mol. Gew. 7815

Pentacabomyl/ 7-4t-butyl-p-tolylmethylenamino)(3,3-o-

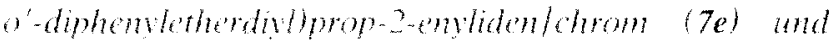


Pentacarbonyl['butyl-p-tolylmethylenamin]chrom. $\mathrm{Zu}$ einer Lösung von $0.9 \mathrm{~g}(2.0 \mathrm{mmol}) 2 \mathrm{e}$ in $\mathrm{CH}_{2} \mathrm{Cl}_{2}$ wird 1 $\mathrm{ml}(6 \mathrm{mmol}){ }^{\text {tButyl }}(p$-tolyl)methylenamin gegeben. Nach Rühren über Nacht bei Raumtemp. zeigt das IR-Spektrum der Lösung keine Eduktbanden mehr. Chromatographie mit Pentan $/ \mathrm{CH}_{2} \mathrm{Cl}_{2}(1 / 1)$ an Kieselgel bei $-20^{\circ} \mathrm{C}$ liefert nach zweimaliger Kristallisation aus $45 \mathrm{ml}$ Pentan/ $\mathrm{Et}_{2} \mathrm{O}(3 / 1) 7 \mathrm{e}$ in Form gelber, filziger Nadeln. Ausb. $0.53 \mathrm{~g} \mathrm{(46 \%}$ bez. auf $2 \mathbf{e}$ ). Schmp. $126^{\circ} \mathrm{C}$. IR (n-Pentan): $\nu$ (CO) 2054w, 1970vw, $1935 \mathrm{v}, 1922 \mathrm{~m} \mathrm{~cm}^{-1}$. ${ }^{1} \mathrm{H}-\mathrm{NMR}$ ( $\mathrm{CDCl}_{3}$, Raumtemp.): $\delta=1.23\left(\mathrm{~s}, 9 \mathrm{H}, \mathrm{C}\left(\mathrm{CH}_{3}\right)_{3}\right) ; 2.41\left(\mathrm{~s}, 3 \mathrm{H}, \mathrm{C}_{6} \mathrm{H}_{4} \mathrm{CH}_{3}\right) ; 6.64$ $(\mathrm{s}, 1 \mathrm{H},=\mathrm{CH}) ; 6.27-7.70(\mathrm{~m}, 12 \mathrm{H}$, Aromaten $) .{ }^{13} \mathrm{C}-\mathrm{NMR}$ $\left(\mathrm{CDCl}_{3}\right.$, Raumtemp.): $\delta=223.0$ (trans-CO); 217.3 (cis$\mathrm{CO}) ; 199.5($ Carben-C); $139.8(\mathrm{~N}=\mathrm{C}) ; 152.0 ; 151.7 ; 131.8$; $129.3 ; 129.1 ; 127.8 ; 127.5 ; 126.7 ; 124.0 ; 123.5 ; 122.9$; $122.8 ; 122.1 ; 121.0 ; 119.4 ; 116.9 ; 116.3$ (Aromaten und $=\mathrm{C}) ; 37.8\left(\mathrm{C}(\mathrm{Me})_{3}\right] ; 28.1\left[\mathrm{C}\left(\mathrm{CH}_{3}\right)_{3}\right] ; 21.3\left(\mathrm{C}_{6} \mathrm{H}_{4} \mathrm{CH}_{3}\right)$. Analyse: Gef.: C, 66.82; $\mathrm{H}, 4.50 ; \mathrm{N}, 2.64 . \mathrm{C}_{32} \mathrm{H}_{25} \mathrm{CrNO}_{6}$ ber.: C, 67.25; H, 4.41; N, 2.45\%; Mol.-Gew. 571.5.

\section{6. ((1-Isopropyl-3-diphenylmethyliden-2-phenyl)-} azetidinyliden]pentacarbonylwolfram (9)

Eine Lösung von nach 5.2.1. aus $5 \mathrm{mmol} \mathrm{W}(\mathrm{CO})_{6}$ dargestelltem $1 \mathrm{a}$ in $20 \mathrm{ml} \mathrm{CH}_{2} \mathrm{Cl}_{2}$ wird bei $-25^{\circ} \mathrm{C}$ mit $5 \mathrm{ml}(30 \mathrm{mmol})$ Benzylidenisopropylamin versetzt und $45 \mathrm{~h}$ bei $-30^{\circ} \mathrm{C}$ gerührt. Die braune Lösung wird zur Trockne gebracht und bei $-20^{\circ} \mathrm{C}$ an Kieselgel chromatographiert. 9 wird mit Pentan $/ \mathrm{CH}_{2} \mathrm{Cl}_{2}$-Gemischen (aufsteigend polar bis 7/3) eluiert. Nach einem violetten Vorlauf (das IR-Spektrum zeigt nur $\mathrm{W}(\mathrm{CO})_{6}$ ) wird eine lange, grünlich durchsetzte gelbe Zone abgetrennt. Nach Entfernen des Lösungsmittels wird der ölige Rückstand bei $-20^{\circ} \mathrm{C}$ an Kieselgel mit Pentan $/ \mathrm{CH}_{2} \mathrm{Cl}_{2}(8 / 2)$ chromatographiert. Die gelbe Produktfraktion wird zur Trockne gebracht, in $30 \mathrm{ml}$ Pentan $/ \mathrm{CH}_{2} \mathrm{Cl}_{2}(8 / 2)$ aufgenommen, mit $20 \mathrm{ml}$ Pentan überschichtet und bei $-30^{\circ} \mathrm{C}$ kristallisiert. 9 wird in Form gelber Kristalle erhalten. Ausb. $320 \mathrm{mg}(10 \%$ bez. auf $\left.\mathrm{W}(\mathrm{CO})_{6}\right)$. Zers. ab $135^{\circ} \mathrm{C}$. IR (n-Pentan): $\nu(\mathrm{CO}) 2061 \mathrm{w}, 1970 \mathrm{vw}, 1927 \mathrm{vs} \mathrm{cm}{ }^{-1} .{ }^{1} \mathrm{H}-\mathrm{NMR}\left(\mathrm{CDCl}_{3}\right.$, Raumtemp.): $\delta=0.99$ (d, $\left.6.8 \mathrm{~Hz}, 3 \mathrm{H}, \mathrm{CH}_{3}\right) ; 1.40$ (d, $\left.6.6 \mathrm{~Hz}, 3 \mathrm{H}, \mathrm{CH}_{3}\right) ; 4.71$ (sept, $6.7 \mathrm{~Hz}, 1 \mathrm{H}, \mathrm{CH}\left(\mathrm{CH}_{3}\right)_{2}$ ); $6.02\left(\mathrm{~s}, 1 \mathrm{H}\right.$, Ring-CH); 6.85-7.48 (m, 15H, Ph). ${ }^{1.3} \mathrm{C}-$ NMR ( $\mathrm{CDCl}_{3}$, Raumtemp.): $\delta=248.5$ (Carben-C); 202.0 (trans-CO); 197.8 (cis-CO, $J(\mathrm{WC}) 126 \mathrm{~Hz}) ; 145.3$; $143.7 ; 139.4 ; 137.3 ; 134.2 ; 131.5 ; 129.5 ; 129.1 ; 128.8$; $128.4 ; 128.3 ; 128.2 ; 127.7 ; 127.4(\mathrm{Ph}+=\mathrm{C}) ; 76.3\left(\mathrm{sp}^{3}-\right.$ Ring-C); $55.2\left[\mathrm{CH}\left(\mathrm{CH}_{3}\right)_{2}\right] ; 22.7\left(\mathrm{CH}_{3}\right) ; 20.5\left(\mathrm{CH}_{3}\right)$. Analyse: Gef.: C, 54.45; H, 3.52; N, 2.30; Mol.-Gew. 661 (EI-MS, ${ }^{184}$ W). $\mathrm{C}_{30} \mathrm{H}_{23} \mathrm{NO}_{5} \mathrm{~W}$ ber.: $\mathrm{C}, 54.48 ; \mathrm{H}$, 3.51; N, 2.12\%; Mol.-Gew. 661.3.

\subsection{Reaktion von Id mit $N, N^{\prime}$-Dimethylhydrazin}

Eine Lösung von $10 \mathrm{mmol}(\mathrm{CO})_{5} \mathrm{~W}[\mathrm{THF}]$ in $160 \mathrm{ml}$ THF wird bei Raumtemp. mit $2.95 \mathrm{~g}$ (10 mmol) 1,1Bis( $p$-dimethylaminophenyl)prop-2-in-1-ol versetzt und 30 min gerührt. Die blaue Lösung wird im Vakuum zur Trockne gebracht und der Rückstand in $50 \mathrm{ml} \mathrm{CH}_{2} \mathrm{Cl}_{2}$ aufgenommen. Nach 30 min wird bei $-10^{\circ} \mathrm{C}$ unter Rühren ein Gemisch aus $1.33 \mathrm{~g}(10 \mathrm{mmol}) N, N^{\prime}$-Dimethylhydrazin-Dihydrochlorid und $1.12 \mathrm{~g}(20 \mathrm{mmol})$ Kaliumhydroxid in $5 \mathrm{ml} \mathrm{H}_{2} \mathrm{O}$ zugegeben. Das Reaktionsgemisch wird $1 \mathrm{~h}$ bei Raumtemp. gerührt und dann in Vakuum zur Trockne gebracht. Der braune Rückstand wird bei $-20^{\circ} \mathrm{C}$ an Kieselgel chromatographiert. Mit Pentan $/ \mathrm{CH}_{2} \mathrm{Cl}_{2} / \mathrm{Et}_{2} \mathrm{O}$-Gemischen (aufsteigend polar bis $6 / 2 / 2$ ) wird nach einem gelben Vorlauf $\left[\mathrm{W}(\mathrm{CO})_{6}\right]$ eine schlecht laufende, orange-gelbe Zone eluiert. Die lange Produktfraktion wird zweimal geschnitten.

Die erste Teilfraktion liefert nach Umkristallisation aus $30 \mathrm{ml}$ Pentan $/ \mathrm{CH}_{2} \mathrm{Cl}_{2}(8 / 2) \mathrm{Z}$-11d $\boldsymbol{\alpha}$ in hellroten Nadeln. Der schlechter laufende Komplex $\boldsymbol{E}$-11d $\boldsymbol{\alpha}$ kristallisiert aus $30 \mathrm{ml}$ Pentan $/ \mathrm{CH}_{2} \mathrm{Cl}_{2}(8 / 2)$ nach Entfernen des Lösungsmittels aus dem dritten Teil der Produktfraktion in Form von orangen Rauten. Der kleinere, mittlere Teil der Produktzone stellt ein Gemisch von $\boldsymbol{E}$-11d $\boldsymbol{\alpha}$ und $Z-11 d \boldsymbol{\alpha}(7 / 5)$ dar.

Pentacarbonyl/(E-1-N,N'-dimethylhydrazino-3,3-bis-pdimethylaminophenyl)prop-2-enyliden Jwolfram (E$11 d \boldsymbol{\alpha})$. Ausb. $1.36 \mathrm{~g}\left(21 \%\right.$ bez. auf $\left.\mathrm{W}(\mathrm{CO})_{6}\right)$. Zers. ab $100^{\circ} \mathrm{C}$. IR $\left(\mathrm{Et}_{2} \mathrm{O}\right): \nu(\mathrm{CO}) 2060 \mathrm{w}, 1968 \mathrm{vw}, 1925 \mathrm{vs} \mathrm{cm} \mathrm{cm}^{-1}$. ${ }^{1} \mathrm{H}-\mathrm{NMR}\left(\mathrm{CDCl}_{3}\right.$, Raumtemp.): $\delta=2.50(\mathrm{~d}, 6.2 \mathrm{~Hz}$, $\left.3 \mathrm{H}, \mathrm{NHCH}_{3}\right) ; 2.97\left(\mathrm{~s}, 6 \mathrm{H}, \mathrm{N}\left(\mathrm{CH}_{3}\right)_{2}\right) ; 2.98(\mathrm{~s}, 6 \mathrm{H}$, $\left.\mathrm{N}\left(\mathrm{CH}_{3}\right)_{2}\right) ; 3.78\left(\mathrm{~s}, 3 \mathrm{H}, \mathrm{N}_{\alpha} \mathrm{CH}_{3}\right) ; 6.26(\mathrm{q}, 6.2 \mathrm{~Hz}, 1 \mathrm{H}$, $\mathrm{NH}) ; 6.45(\mathrm{~s}, 1 \mathrm{H},=\mathrm{CH}) ; 6.60-7.24(\mathrm{~m}, 8 \mathrm{H}$, Aromaten $)$. ${ }^{13} \mathrm{C}-\mathrm{NMR}\left(\mathrm{CDCl}_{3}\right.$, Raumtemp.): $\delta=231.9$ (Carben-C); 203.3 (trans-CO); 198.4 (cis-CO, J(WC) $127.4 \mathrm{~Hz}$ ); $135.5\left[=C(\text { Aryl })_{2}\right] ; 129.7(=\mathrm{CH}) ; 150.4 ; 150.3 ; 130.9$; $129.9 ; 129.0 ; 125.7 ; 111.9 ; 111.6$ (Aromaten); 48.2 $\left.\left(\mathrm{N}_{\alpha} \mathrm{CH}_{3}\right) ; 40.4 \quad\left(\mathrm{~N}^{2}\left(\mathrm{CH}_{3}\right)_{2}\right) ; 40.1 \quad\left(\mathrm{~N}^{2} \mathrm{CH}_{3}\right)_{2}\right) ; 36.1$ $\left(\mathrm{NHCH}_{3}\right)$. Analyse: Gef.: C, 48.59; $\mathrm{H}, 4.86 ; \mathrm{N}, 8.10$; Mol.-Gew. 660 (EI-MS, ${ }^{184} \mathrm{~W}$ ). $\mathrm{C}_{26} \mathrm{H}_{28} \mathrm{~N}_{4} \mathrm{O}_{5} \mathrm{~W} \cdot 1 / 3$ $\mathrm{C}_{5} \mathrm{H}_{12}$ ber.: C, 48.55; H, 4.71; N, 8.19\%; Mol.-Gew. $660.4+24.1(\boldsymbol{E}$-11d $\boldsymbol{\alpha}$ konnte bislang nicht vollständig lösungsmittelfrei erhalten werden.)

Pentacarbonyl/(Z-1-N,N'-dimethylhydrazino-3,3-bis-pdimethylaminophenyl)prop-2-enyliden/wolfram (Z$11 d \alpha)$. Ausb. $1.23 \mathrm{~g}\left(19 \%\right.$ bez. auf $\left.\mathrm{W}(\mathrm{CO})_{6}\right)$. Schmp. $86-87^{\circ} \mathrm{C}$ (Zers.). IR $\left(\mathrm{Et}_{2} \mathrm{O}\right): \nu(\mathrm{CO}) 2061 \mathrm{w}, 1971 \mathrm{ww}$, $1925 \mathrm{vs} \mathrm{cm}^{-1} .{ }^{1} \mathrm{H}-\mathrm{NMR}\left(\mathrm{CDCl}_{3}\right.$, Raumtemp.): $\delta=2.68$ $\left(\mathrm{d}, 6.3 \mathrm{~Hz}, 3 \mathrm{H}, \mathrm{NHCH} \mathrm{H}_{3}\right) ; 2.96\left(\mathrm{~s}, 6 \mathrm{H}, \mathrm{N}\left(\mathrm{CH}_{3}\right)_{2}\right) ; 2.98$ $\left(\mathrm{s}, 6 \mathrm{H}, \mathrm{N}\left(\mathrm{CH}_{3}\right)_{2}\right) ; 3.10\left(\mathrm{~s}, 3 \mathrm{H}, \mathrm{N}_{\alpha} \mathrm{CH}_{3}\right) ; 6.15(\mathrm{q}, 6.3 \mathrm{~Hz}$, $1 \mathrm{H}, \mathrm{NH}) ; 6.57(\mathrm{~s}, 1 \mathrm{H},=\mathrm{CH}) ; 6.60-7.24(\mathrm{~m}, 8 \mathrm{H}$, Aromaten). ${ }^{13} \mathrm{C}$-NMR $\left(\mathrm{CDCl}_{3}\right.$, Raumtemp.): $\delta=227.6$ 
(Carben-C); 202.5 (trans-CO; 197.5 (cis-CO, J(WC) $128 \mathrm{~Hz}) ; 150.3 ; 150.1$ (Aromaten); $136.3\left[=\mathrm{C}(\text { Aryl })_{2}\right]$; $131.8(=\mathrm{CH}) ; 130.8 ; 130.0 ; 129.8 ; 126.9 ; 111.8 ; 111.6$ (Aromaten); $40.4 \quad\left(\mathrm{~N}\left(\mathrm{CH}_{3}\right)_{2}\right) ; 40.3 \quad\left(\mathrm{~N}\left(\mathrm{CH}_{3}\right)_{2}\right) ; 39.5$ $\left(\mathrm{N}_{2} \mathrm{CH}_{3}\right) ; 35.8\left(\mathrm{NHCH}_{3}\right)$. Analyse: Gef.: $\mathrm{C}, 47.39 ; \mathrm{H}$, 4.34; N, 8.45; Mol.-Gew. 660 (EI-MS, ${ }^{184} \mathrm{~W}$ ). $\mathrm{C}_{26} \mathrm{H}_{28} \mathrm{~N}_{4} \mathrm{O}_{5} \mathrm{~W}$ ber: $\mathrm{C} .47 .29: \mathrm{H}, 4.27 ; \mathrm{N}, 8.48 \% ;$ Mol.Gew. 660.4.

\subsection{Reaktion ion Id mit 1-Methyl-2-phenylhydrazin}

Eine Lösung von $5 \mathrm{mmol}(\mathrm{CO})_{5} \mathrm{~W}[\mathrm{THF}]$ in $80 \mathrm{ml}$ THF wird bei Raumtemp. mit $1.47 \mathrm{~g}(5 \mathrm{mmol}), 1,1$ Bis( $p$-dimethylaminophenyl)prop-2-in-1-ol versetzt und 30 min gerührt. Die blaue Lösung wird im Vakuum zur Trockne gebracht und der Rückstand in $50 \mathrm{ml} \mathrm{CH}_{2} \mathrm{Cl}_{2}$ aufgenommen. Nach $30 \mathrm{~min}$ werden bei $0^{\circ} \mathrm{C} 0.6 \mathrm{~g}(5$ mmol) MeNH-HNPh zugegeben und $6 \mathrm{~h}$ bei Raumtemp. gerührt. Das Reaktionsgemisch wird im Vakuum zur Trockne gebracht und bei $-20^{\circ} \mathrm{C}$ an Kieselgel chromatographiert. Die Reaktionsprodukte werden mit Pentan $/ \mathrm{CH}_{2} \mathrm{Cl}_{2} / \mathrm{Et}_{2} \mathrm{O}$-Gemischen (aufsteigend polar bis $7 / 2 / 1$ ) eluiert. Es kann zunächst eine orange Zone von $\boldsymbol{E}$-11d $\boldsymbol{\beta}$ abgetrennt werden. Nachfolgend läuft das gelb-orange $Z$-11d $\boldsymbol{\beta}, \boldsymbol{E}$-11d $\boldsymbol{\beta}$ kristallisiert aus $20 \mathrm{ml}$ Pentan $/ \mathrm{CH}_{2} \mathrm{Cl}_{2}(9 / 1)$ in orangen Kristallen. $\boldsymbol{Z}$-11d $\boldsymbol{\beta}$ kristallisiert aus $25 \mathrm{ml}$ Pentan $/ \mathrm{CH}_{2} \mathrm{Cl}_{2}(9 / 1)$ in gelborangen Kristallen.

Pentacarbonyl/ (E-I-No-methyl-N $N_{\beta}$-phenylhydrazino-3,3bis-p-dimethylaminophenyl)prop-2-enyliden/wolfram $(\boldsymbol{E}$ IId $\boldsymbol{\beta})$. Ausb. $0.94 \mathrm{~g}\left(26 \%\right.$ bez. auf $\left.\mathrm{W}(\mathrm{CO})_{6}\right)$. Zers. ab $110^{\circ} \mathrm{C}$. IR (Et $\left.{ }_{2} \mathrm{O}\right): \nu(\mathrm{CO}) 2060 \mathrm{w}, 1970 \mathrm{ww}, 1929 \mathrm{v}$ $1914 \mathrm{msh} \mathrm{cm}^{1}{ }^{1} \mathrm{H}-\mathrm{NMR}\left(\mathrm{CDCl}_{3}\right.$, Raumtemp.): $\delta=$ $2.93\left(\mathrm{~s}, 6 \mathrm{H}, \mathrm{N}\left(\mathrm{CH}_{3}\right)_{2}\right) ; 3.05\left(\mathrm{~s}, 6 \mathrm{H}, \mathrm{N}\left(\mathrm{CH}_{3}\right)_{2}\right) ; 3.93(\mathrm{~d}$. $\left.0.9 \mathrm{~Hz}, 3 \mathrm{H}, \mathrm{N}_{\alpha} \mathrm{CH}_{3}\right) ; 6.18(\mathrm{~s}, 1 \mathrm{H}, \mathrm{NH}) ; 6.35(\mathrm{~s}, 1 \mathrm{H}$, $=\mathrm{CH}) ; \quad 6.36-7.29\left(\mathrm{~m}, 13 \mathrm{H}\right.$, Aromaten), ${ }^{13} \mathrm{C}-\mathrm{NMR}$ ( $\mathrm{CDCl}_{3}$, Raumtemp.): $\delta=247.7$ (Carben-C); 203.9 (trans-CO); 198.4 (cis-CO, J(WC) $127 \mathrm{~Hz}) ; 134.3$ $\left[=C(\text { Aryl })_{2}\right] ; 132.9 \quad(=\mathrm{CH}) ; 150.7 ; 150.2,144.8 ; 130.3 ;$ $130.2 ; 129.7 ; 129.4 ; 126.2 ; 121.5 ; 112.8 ; 112.1 ; 111.5$ (Aromaten); $52.8 \quad\left(\mathrm{~N}_{c x} \mathrm{CH}_{3}\right) ; 40.3 \quad\left(\mathrm{~N}\left(\mathrm{CH}_{3}\right)_{2}\right) ; 40.2$ $\left(\mathrm{N}\left(\mathrm{CH}_{3}\right)_{2}\right)$. Analyse: Gef.: $\mathrm{C}, 51.27 ; \mathrm{H}, 4.17 ; \mathrm{N}, 7.60$. $\mathrm{C}_{31} \mathrm{H}_{30} \mathrm{~N}_{4} \mathrm{O}_{5} \mathrm{~W}$ ber.: C, 51.54; H, 4.19; N, 7.76\%; Mol.Gew. 722.4.

Pentacarbonyl/(Z-1-N - methyl-N - phenylhydrazino-3,3bis-p-dimethylaminophenyl)prop-2-enyliden/wolfram (Z11d $\boldsymbol{\beta})$. Ausb. $1.01 \mathrm{~g}\left(28 \%\right.$ bez. auf $\left.\mathrm{W}(\mathrm{CO})_{6}\right)$. Zers. ab $110^{\circ} \mathrm{C}$. IR (Et $\left.{ }_{2} \mathrm{O}\right): \nu(\mathrm{CO}) 2058 \mathrm{w}, 1968 \mathrm{ww}, 1925 \mathrm{vs}$, $1909 \mathrm{msh} \mathrm{cm}^{-1}$. 'H-NMR ( $\mathrm{CDCl}_{3}$, Raumtemp.): $\delta=$ $2.98\left(\mathrm{~s}, 6 \mathrm{H}, \mathrm{N}\left(\mathrm{CH}_{3}\right)_{2}\right) ; 3.02\left(\mathrm{~s}, 6 \mathrm{H}, \mathrm{N}\left(\mathrm{CH}_{3}\right)_{2}\right) ; 3.27(\mathrm{~s}$, $\left.3 \mathrm{H}, \mathrm{N}_{\alpha} \mathrm{CH}_{3}\right) ; 6.56-6.75(\mathrm{~m}, 6 \mathrm{H}$, Aromaten); $6.79(\mathrm{~s}$, $1 \mathrm{H},=\mathrm{CH}) ; 6.98-7.25(\mathrm{~m}, 7 \mathrm{H}$, Aromaten $) ; 7.14(\mathrm{~s}, 1 \mathrm{H}$, NH). ${ }^{13} \mathrm{C}-\mathrm{NMR} \quad\left(\mathrm{CDCl}_{3}\right.$, Raumtemp.): $\delta=245.7$ (Carben-C); 203.3 (trans-CO); 197.8 (cis-CO, J(WC)
$127 \mathrm{~Hz}) ; 136.1\left[=\mathrm{C}(\text { Aryl })_{2}\right] ; 132.2(=\mathrm{CH}) ; 150.4 ; 150.1 ;$ $142.9 ; 130.8 ; 129.7 ; 129.6 ; 129.4 ; 127.2 ; 123.3 ; 116.4 ;$ 112.0; 111.8 (Aromaten): $43.1 \quad\left(\mathrm{~N}_{4} \mathrm{CH}_{3}\right) ; 40.4 \quad(\mathrm{~N}$ $\left.\left(\mathrm{CH}_{3}\right)_{2}\right) ; 40.3\left(\mathrm{~N}\left(\mathrm{CH}_{3}\right)\right.$ ). Analyse: Gef: $\mathrm{C} .51 .27 ; \mathrm{H}$, $4.07 ; \mathrm{N}, 7.59 . \mathrm{C}_{31} \mathrm{H}_{30} \mathrm{~N}_{4} \mathrm{O}, \mathrm{W}$ ber.: $\mathrm{C}, 51.54: \mathrm{H}, 4.19 ; \mathrm{N}$. $7.76 \%$; Mol.-Gew. 722,4

5.9. Reaktionen lon Ic und Id mit Hydrazin und Phenylhydrazin 1(2.2-Bis-p-methoxyphenyl)acrylnitrill(pentacarbonyl)wolfram (12c). Eine nach 5.2 .1 aus $5 \mathrm{mmol}$ $W(\mathrm{CO})_{6}$ dargestellte Lösung von $1 \mathrm{c}$ in $20 \mathrm{ml} \mathrm{CH} \mathrm{Cl}_{2}$ wird bei $-25^{\circ} \mathrm{C}$ mit $0.47 \mathrm{ml}$ Hydrazinhydrat $64 \%$ ige Lösung; 6 mmol) versetzt. Nach $30 \mathrm{~min}$ Rühren bei Raumtemp. wird das Lösungsmittel entfernt und der grüne Rückstand bei $-20^{\circ} \mathrm{C}$ an Kieselgel chromatographiert. 12c wird zuerst mit Pentan, danach mit Pen$\tan / \mathrm{CH}, \mathrm{Cl}$, (aufsteigend polar bis $4 / 6$ ) als vorauslaufende, gelbe Zone eluiert. Unkristallisation aus $25 \mathrm{ml}$ Pentan $/ \mathrm{CH}_{2} \mathrm{Cl}_{2}(9 / 1)$ liefent $12 \mathrm{c}$ als gelbe $\mathrm{Nadeln}$. Ausb. $410 \mathrm{mg}\left(14 \%\right.$ bez. auf $\left.\mathrm{W}(\mathrm{CO})_{0}\right)$. Schmp. $128-$ $129^{\circ} \mathrm{C}$ (Zers.). IR (n-Pentan): $r(\mathrm{CO}) 2072 \mathrm{w}, 1943 \mathrm{vs}$. $1922 \mathrm{~m} \mathrm{~cm}^{-1}$ 'H-NMR (CDCl ; Raumtemp.): $\delta=3.85$ $\left(\mathrm{s}, 3 \mathrm{H}, \mathrm{OCH}_{3}\right) ; 3.87(\mathrm{~s}, 3 \mathrm{H}, \mathrm{OCH}, ; 5.61(\mathrm{~s}, 1 \mathrm{H},=\mathrm{CH})$; 6.85-7.38 (m, 8H. Aromaten). "C-NMR (CDCl 3 , Raumtemp.): $\dot{\partial}=200.4$ (trans-CO); 196.2 (cis-CO. J(WC) 130 Hz); $166.7\left[=C(\mathrm{Aryl})_{2}\right] ; 162.3 ; 161.7 ; 131.2 ;$ 130.6; 130.5; 129.0; 114.2 (Aromaten); $125.0(\mathrm{~N} \equiv \mathrm{C})$; $88.9(=\mathrm{CH}) ; 55.5(\mathrm{OCH}), 55.4\left(\mathrm{OCH}_{3}\right)$. Analyse: Gef: $\mathrm{C}, 44.76 ; \mathrm{H}, 2.62 ; \mathrm{N} .2 .42 . \mathrm{C}_{22} \mathrm{H}_{15} \mathrm{NO}_{7} \mathrm{~W}$ ber.: $\mathrm{C}, 44.84 ;$ H. 2.57: N. 2.38\%: Mol.-Gew. 589.2.

[(2,2-Bis-p-dimethylaminophenyl)acrylnitrill(pentacar bonyl)wolfram (12d).

Aus Hydrazin: $180 \mathrm{mg}(0.3 \mathrm{mmol})$ 1d werden in 10 $\mathrm{ml} \mathrm{CH} \mathrm{Cl}_{2}$ gelöst und bei Raumtemp. mit $0.03 \mathrm{ml}$ Hydrazinhydrat (64\%ige Lösung: $0.4 \mathrm{mmol}$ ) versetzt. Nach 15 min wird das Lösungsmittel entfernt und der Rückstand bei Raumtemp. an Kieselgel chromatographiert. 12d läuft bei der Elution mit Pentan $/ \mathrm{CH}_{2} \mathrm{Cl}_{2}$ $(4 / 3)$ als gelbe Zone voraus und kristallisiert nach Entfernung des Lösungsmittels aus $10 \mathrm{ml}$ Penlan $/ \mathrm{CH}_{2} \mathrm{Cl}_{2}(8 / 2)$ in Form von gelben Nadeln. Ausb. $80 \mathrm{mg}$ (439 bez. auf Id). Schmp. $144-147^{\circ} \mathrm{C}$ (Zers.).

Aus Phenylhydrazin: $1.04 \mathrm{~g}(1.73 \mathrm{mmol}) 1 \mathrm{~d}$ werden in $20 \mathrm{ml} \mathrm{CH} \mathrm{Cl}_{2}$ gelöst und bei Raumtemp. mit 0.30 $\mathrm{ml}(3 \mathrm{mmol})$ Phenyllydrazin versetzt. Nach 8 h Rühren wird die grüne Lösung im Vakuum zur Trockne gebracht und der Rückstand bei $-20^{\circ} \mathrm{C}$ an Kieselgel chromatographiert. 12d läuft bei der Elution mit Pen$\tan / \mathrm{Et}_{2} \mathrm{O}$-Gemischen (aufsteigend polar bis $1 / 1$ ) als gelbe Zone voraus. Nach Entfernen des Lösungsmittels kristallisiert $12 \mathrm{~d}$ aus $20 \mathrm{ml}$ Pentan $/ \mathrm{CH}_{2} \mathrm{Cl},(8 / 2)$ in Form von getben Nadeln. Aush. $395 \mathrm{mg}(37 \%$, hez. auf 
1d). Schmp. $145-149^{\circ} \mathrm{C}$ (Zers.). IR (n-Pentan): $\nu$ (CO) 2072w, 1941vs, 1939vssh, 1918s cm ${ }^{-1}$. ${ }^{1} \mathrm{H}-\mathrm{NMR}$ $\left(\mathrm{CDCl}_{3}\right.$, Raumtemp.): $\delta=3.04\left(\mathrm{~s}, 12 \mathrm{H}, \mathrm{N}\left(\mathrm{CH}_{3}\right)_{2}\right) ; 5.37$ $(\mathrm{s}, 1 \mathrm{H},=\mathrm{CH}) ; 6.62-7.35\left(\mathrm{~m}, 8 \mathrm{H}\right.$, Aromaten). ${ }^{13} \mathrm{C}-\mathrm{NMR}$ $\left(\mathrm{CDCl}_{3}\right.$, Raumtemp.): $\delta=200.8$ (trans-CO); 196.5 (cis$\mathrm{CO}, J(\mathrm{WC}) 130 \mathrm{~Hz}) ; 167.4\left[=C(\text { Aryl })_{2}\right] ; 152.2 ; 152.0$; $131.2 ; 130.6 ; 126.8 ; 125.7 ; 111.4$ (Aromaten); 124.1 $(\mathrm{N} \equiv \mathrm{C}) ; 83.7(=\mathrm{CH}) ; 40.1\left(\mathrm{~N}\left(\mathrm{CH}_{3}\right)_{2}\right) ; 40.0\left(\mathrm{~N}\left(\mathrm{CH}_{3}\right)_{2}\right)$. Analyse: Gef.: C, 46.89; H, 3.43; N, 6.83; Mol.-Gew. 615 (FAB-MS (NBOH), ${ }^{184} \mathrm{~W}$ ). $\mathrm{C}_{24} \mathrm{H}_{21} \mathrm{~N}_{3} \mathrm{O}_{5} \mathrm{~W}$ ber.: $\mathrm{C}$, 46.85; H, 3.44; N, 6.83\%; Mol.-Gew. 615.3.

\subsection{Reaktion von la und 1 d mit $N, N$-Dimethylhydrazin} Pentacarbonyl [(1-dimethylamino-3,3-diphenyl)prop-2enyliden/wolfram (13a) und Pentacarbonyll(2,2-diphenyl)acrylnitril/wolfram (12a). Eine Lösung von nach 5.2.1. aus $5 \mathrm{mmol} \mathrm{W}(\mathrm{CO})_{6}$ dargestelltem $1 \mathrm{a}$ in $25 \mathrm{ml}$ $\mathrm{CH}_{2} \mathrm{Cl}_{2}$ wird bei $-45^{\circ} \mathrm{C}$ mit $0.38 \mathrm{ml}(5 \mathrm{mmol}) N, N$-Dimethylhydrazin versetzt. Man lässt die Lösung über Nacht bei $-30^{\circ} \mathrm{C}$ stehen und erwärmt dann auf Raumtemp. Das braune Reaktionsgemisch wird im Vakuum zur Trockne gebracht und bei $-20^{\circ} \mathrm{C}$ an Kieselgel chromatographiert. Es wird zuerst mit Pentan, dann mit Pentan $/ \mathrm{CH}_{2} \mathrm{Cl}_{2} / \mathrm{Et}_{2} \mathrm{O}$ (aufsteigend polar bis $8 / 1 / 1 /$ ) eluiert. Nach einem gelb-braunen Vorlauf $\left[\mathrm{W}(\mathrm{CO})_{6}\right]$ kann eine gelbe Zone, die 12a enthält, abgetrennt werden. Nachfolgend läuft 13a als weitere gelbe Fraktion. Beide Fraktionen werden im Vakuum zur Trockne gebracht. 12a kristallisiert aus $10 \mathrm{ml}$ Pen$\tan / \mathrm{CH}_{2} \mathrm{Cl}_{2}(10 / 1)$ in gelben Nadeln. Umkristallisation aus $10 \mathrm{ml}$ Pentan $/ \mathrm{CH}_{2} \mathrm{Cl}_{2}(10 / 1)$ liefert 13a als gelbe Kristalle.

12a: Ausb. $80 \mathrm{mg}$ (3\% bez. auf $\mathrm{W}(\mathrm{CO})_{6}$ ). Schmp. $84-86^{\circ} \mathrm{C}$. IR (n-Pentan): $\nu$ (CO) $2072 \mathrm{w}, 1945 \mathrm{vs}, 1925 \mathrm{~m}$ $\mathrm{cm}^{-1} .{ }^{1} \mathrm{H}-\mathrm{NMR}\left(\mathrm{CDCl}_{3}\right.$, Raumtemp.): $\delta=5.83(\mathrm{~s}, 1 \mathrm{H}$, $=\mathrm{CH}) ; 7.31-7.60(\mathrm{~m}, 10 \mathrm{H}, \mathrm{Ph}) .{ }^{13} \mathrm{C}-\mathrm{NMR}\left(\mathrm{CDCl}_{3}\right.$, Raumtemp.): $\delta=200.2$ (trans-CO); 196.1 (cis-CO); $167.4\left(=\mathrm{CPh}_{2}\right) ; 137.9 ; 136.6 ; 131.3 ; 130.9 ; 129.3 ; 128.9$; $128.7(\mathrm{Ph}) ; 123.9(\mathrm{~N} \equiv \mathrm{C}) ; 92.7(=\mathrm{CH})$. Analyse: Gef.: $\mathrm{C}$, $45.44 ; \mathrm{H}, 2.16 ; \mathrm{N}, 2.80 . \mathrm{C}_{2 n} \mathrm{H}_{11} \mathrm{NO}_{5} \mathrm{~W}$ ber.: $\mathrm{C}, 45.39 ; \mathrm{H}$, $2.10 ; \mathrm{N}, 2.65 \%$; Mol.-Gew. 529.1 .

13a: Ausb. $70 \mathrm{mg}(2.5 \% \text { bez. auf W(CO) })_{6}$. Schmp. $130-131^{\circ} \mathrm{C}$. Analyse: Gef.: C, 47.24; H, 3.07; N, 2.68; Mol.-Gew. 559 (EI-MS, ${ }^{184} \mathrm{~W}$ ). $\mathrm{C}_{22} \mathrm{H}_{17} \mathrm{NO}_{5} \mathrm{~W}$ ber.: $\mathrm{C}$, 47.25; H, 3.06; N, 2.50\%; Mol.-Gew. 559.2. Alle spektroskopischen Daten von 13a stimmen mit den Literaturwerten überein [30].

Pentacarbonyl/(1-dimethylamino-3,3-bis-p-dimethylaminophenyl)prop-2-enyliden/wolfram (13d) und Pentacarbonyl/(2,2-bis-p-dimethylaminophenyl)acrylnitril]wolfram (12d). $300 \mathrm{mg}(0.5 \mathrm{mmol})$ 1d werden in $10 \mathrm{ml} \mathrm{CH} \mathrm{Cl}_{2}$ gelöst und bei Raumtemp. mit $0.08 \mathrm{ml}(1 \mathrm{mmol}) \mathrm{N}, \mathrm{N}$ Dimethylhydrazin versetzt. Es wird $1.5 \mathrm{~h}$ gerührt und danach das Lösungsmittel entfernt. Der braune
Rückstand wird bei $-20^{\circ} \mathrm{C}$ an Kieselgel chromatographiert. Es wird zuerst mit Pentan, danach mit Pentan $/ \mathrm{CH}_{2} \mathrm{Cl}_{2} / \mathrm{Et}_{2} \mathrm{O}$ (aufsteigend polar bis $6 / 2 / 2$ ) eluiert. Die gelbe Zone mit 12d läuft voraus. Nachfolgend kann die gelbe 13d-Fraktion abgetrennt werden. 13d wird aus $15 \mathrm{ml}$ Pentan $/ \mathrm{CH}_{2} \mathrm{Cl}_{2}(8 / 2)$ in Form von gelben Kristallen erhalten. 12d wird analog zu 5.9: 12d aufgearbeitet und zeigt die identischen spektroskopischen und analytischen Daten. Ausb. $50 \mathrm{mg}$ (16\% bez. auf 1d).

13d: Ausb. $65 \mathrm{mg}$ (20\% bez. auf 1d). Zers. ab $145^{\circ} \mathrm{C}$. IR (n-Pentan): $\nu(\mathrm{CO}) 2060 \mathrm{w}, 1967 \mathrm{vw}, 1931 \mathrm{~s}, 1923 \mathrm{vs}$ $\mathrm{cm}^{-1} .{ }^{1} \mathrm{H}$-NMR $\left(\mathrm{CDCl}_{3}\right.$, Raumtemp.): $\delta=2.94$ (s, 3H, $\left.\mathrm{C}_{\alpha} \mathrm{N}\left(\mathrm{CH}_{3}\right)_{2}\right) ; 2.97\left(\mathrm{~s}, 6 \mathrm{H}, \mathrm{C}_{6} \mathrm{H}_{4} \mathrm{~N}\left(\mathrm{CH}_{3}\right)_{2}\right) ; 2.98(\mathrm{~s}, 6 \mathrm{H}$, $\left.\mathrm{C}_{6} \mathrm{H}_{4} \mathrm{~N}\left(\mathrm{CH}_{3}\right)_{2}\right) ; 3.61\left(\mathrm{~d}, 0.8 \mathrm{~Hz}, 3 \mathrm{H}, \mathrm{C}_{\alpha} \mathrm{N}\left(\mathrm{CH}_{3}\right)_{2}\right)$; $6.68(\mathrm{~s}, 1 \mathrm{H},=\mathrm{CH}) ; 6.59-7.25\left(\mathrm{~m}, 8 \mathrm{H}\right.$, Aromaten). ${ }^{13} \mathrm{C}-$ NMR $\left(\mathrm{CDCl}_{3}\right.$, Raumtemp.): $\delta=248.7$ (Carben-C); 203.9 (trans-CO); 198.7 (cis-CO, J(WC) $127 \mathrm{~Hz}$ ); 150.2; 149.8 (Aromaten); $134.4 \quad(=\mathrm{CH}) ; 133.8\left[=C(\text { Aryl })_{2}\right]$; 130.7; 130.2; 129.8; 127.4; 111.9; 111.6 (Aromaten); 52.7 $\left(\mathrm{C}_{\alpha} \mathrm{N}\left(\mathrm{CH}_{3}\right)_{2}\right) ; 44.2 \quad\left(\mathrm{C}_{\alpha} \mathrm{N}\left(\mathrm{CH}_{3}\right)_{2}\right) ; 40.4 \quad\left(\mathrm{C}_{6} \mathrm{H}_{4}-\right.$ $\left.\mathrm{N}\left(\mathrm{CH}_{3}\right)_{2}\right) ; 40.3\left(\mathrm{C}_{6} \mathrm{H}_{4} \mathrm{~N}\left(\mathrm{CH}_{3}\right)_{2}\right)$. Analyse: Gef.: C, 48.14; H, 4.20; N, 6.60; Mol.-Gew. 645 (EI-MS, ${ }^{184} \mathrm{~W}$ ). $\mathrm{C}_{26} \mathrm{H}_{27} \mathrm{~N}_{3} \mathrm{O}_{5} \mathrm{~W}$ ber.: C, 48.38; H, 4.22; N, 6.50\%; Mol.Gew. 645.3.

\subsection{Reaktion von $\mathbf{I b}$ mit Benzophenonhydrazon $z u$} Pentacarbonyl[(2,2-bis-p-tolyl)acrylnitril]wolfram (12b) und Benzophenonimin (pentacarbonyl)wolfram

Eine Lösung von nach 5.2 .1 aus $5 \mathrm{mmol} \mathrm{W}(\mathrm{CO})_{6}$ dargestelltem $1 \mathbf{b}$ in $25 \mathrm{ml} \mathrm{CH} \mathrm{Cl}_{2}$ wird bei $-40^{\circ} \mathrm{C}$ mit $1.18 \mathrm{~g}(6 \mathrm{mmol})$ Benzophenonhydrazon versetzt. Nach wenigen Sekunden färbt sich die Lösung braun. Das IR-Spektrum des Reaktionsgemisches ändert sich auch durch $16 \mathrm{~h}$ Stehen bei $-30^{\circ} \mathrm{C}$ nicht mehr. Daher wird nach Erwärmen auf Raumtemp. das Lösungsmittel entfernt und der Rückstand bei $-20^{\circ} \mathrm{C}$ an Kieselgel chromatographiert. Es wird zuerst mit Pentan, danach mit Pentan $/ \mathrm{CH}_{2} \mathrm{Cl}_{2}$ (aufsteigend polar bis $8 / 2$ ) eluiert. Nach einem violetten Vorlauf [W(CO) $\left.)_{6}\right]$ kann die gelbe Zone von 12b abgetrennt werden. Anschliessend eluiert man eine orangegelbe Fraktion, die Benzophenonimin(pentacarbonyl)wolfram liefert (Ausb. $195 \mathrm{mg}(8 \% \mathrm{bez}$. auf $\left.\mathrm{W}(\mathrm{CO})_{6}\right)$. Schmp. $91-92^{\circ} \mathrm{C}$. Charakterisierung durch spektroskopischen Vergleich mit Literaturdaten [29]). 12b kristallisiert aus $10 \mathrm{ml}$ Pentan $/ \mathrm{CH}_{2} \mathrm{Cl}_{2}(8 / 2)$ in Form von gelben Nadeln. Ausb. $335 \mathrm{mg}(12 \% \mathrm{bez}$. auf $\mathrm{W}(\mathrm{CO})_{6}$ ). Schmp. $103-105^{\circ} \mathrm{C}$ (Zers.). IR (n-Pen$\tan ): \nu(\mathrm{CO}) 2072 \mathrm{w}, 1944 \mathrm{vs}, 1923 \mathrm{~m} \mathrm{~cm}^{-1}$. ${ }^{1} \mathrm{H}-\mathrm{NMR}$ $\left(\mathrm{CDCl}_{3}\right.$, Raumtemp.): $\delta=2.40\left(\mathrm{~s}, 3 \mathrm{H}, \mathrm{CH}_{3}\right) ; 2.44$ (s, $\left.3 \mathrm{H}, \mathrm{CH}_{3}\right) ; 5.74(\mathrm{~s}, 1 \mathrm{H},=\mathrm{CH}) ; 7.16-7.36(\mathrm{~m}, 8 \mathrm{H}$, Aromaten). ${ }^{13} \mathrm{C}$-NMR $\left(\mathrm{CDCl}_{3}\right.$, Raumtemp.): $\delta=200.4$ (trans-CO); 196.2 (cis-CO, J(WC) $130 \mathrm{~Hz}$ ); 167.6 $\left[=C(\mathrm{Aryl})_{2}\right] ; 142.0 ; 141.4 ; 135.2 ; 133.9 ; 129.6 ; 129.5$; 
TABELLE 6. Kristallstrukturdaten für 6d, $E$-11d $\alpha$ und 12d

\begin{tabular}{|c|c|c|c|}
\hline & $6 d$ & $E-11 \mathrm{~d} \alpha$ & $12 \mathrm{~d}$ \\
\hline Summenformel & $\mathrm{C}_{37} \mathrm{H}_{31} \mathrm{~N}_{3} \mathrm{O}_{5} \mathrm{~W}$ & $\mathrm{C}_{26} \mathrm{H}_{28} \mathrm{~N}_{4} \mathrm{O}_{5} \mathrm{~W} \cdot 0.5 \mathrm{C}_{5} \mathrm{H}_{12}$ & $\mathrm{C}_{24} \mathrm{H}_{2} \mathrm{~N}_{2} \mathrm{O}_{3} \mathrm{~W} \cdot 0.5 \mathrm{CH}_{2} \mathrm{Cl}_{2}$ \\
\hline Molmasse & 781.5 & 696.5 & 657.7 \\
\hline Kristallgrösse $(0.1 \mathrm{~mm})$ & $3 \times 3 \times 3$ & $3 \times 3 \times 3$ & $1 \times 1 \times 2$ \\
\hline Kristallsystem & triklin & monoklin & monoklin \\
\hline Raumgruppe & $P \overrightarrow{1}$ & $P 2_{1} / n$ & $\mathrm{COC}$ \\
\hline$a(\AA)$ & $9.649(3)$ & $11.900(3)$ & $23.12400)$ \\
\hline$b(\AA)$ & $10.42+(3)$ & $9.934(3)$ & $0.88502)$ \\
\hline$c(\AA)$ & $17.262(6)$ & $25.437(8)$ & $32.818(9)$ \\
\hline$\alpha()^{\circ}$ & $103.12(3)$ & 90 & 90 \\
\hline$\beta(5)$ & $93.55(.3)$ & $95.81 \times(2)$ & 94.2602 \\
\hline$\gamma\left({ }^{\circ}\right)$ & $94.23(3)$ & 90 & 90 \\
\hline$V\left(\AA^{3}\right)$ & $1680.8(10)$ & $2991.7(16)$ & $5188(2)$ \\
\hline$Z$ & 2 & 4 & $s$ \\
\hline$d_{\text {her }}\left(\mathrm{g} / \mathrm{cm}^{3}\right)$ & 1.544 & 1.533 & 1682 \\
\hline$\mu(\operatorname{MoK} \alpha)\left(\mathrm{mm}^{-1}\right)$ & 3553 & 3.983 & 4.689 \\
\hline$F(000)(\mathrm{e})$ & 776 & 1364 & 2560 \\
\hline$T(\mathrm{~K})$ & 243 & 243 & 243 \\
\hline$J_{\omega}(0)$ & 0.7 & 0.6 & 0.8 \\
\hline unabh. Reflexe & 0.579 & 6519 & 5671 \\
\hline Reflexe mit $I>3 \sigma$ & 6053 & 5086 & 3817 \\
\hline LS-Parameter & 415 & 334 & 300 \\
\hline$R$ & 0.0280 & 0.0432 & 0.1527 \\
\hline$R_{\mathrm{w}}$ & 0.0364 & 0.0481 & 0.0504 \\
\hline Restelektronendichte $\left(\mathrm{e} / \AA^{3}\right)$ & 1.16 & 1.68 & 1.46 \\
\hline
\end{tabular}

$129.3 ; 128.7$ (Aromaten); $124.4(\mathrm{~N} \equiv \mathrm{C}) ; 91.1(=\mathrm{CH}) ; 21.4$ $\left(\mathrm{CH}_{3}\right)$. Analyse: Gef.: C, 47.53; H, 2.77; N, 2.45. $\mathrm{C}_{22} \mathrm{H}_{15} \mathrm{NO}_{5} \mathrm{~W}$ ber.: $\mathrm{C}, 47.42 ; \mathrm{H}, 2.71 ; \mathrm{N}, 2.51 \%$; Mol.Gew. 557.2.

5.12. Röntgenstrukturanalysen con $\boldsymbol{\sigma d}$, E-11da und 12d (c.gl. Tab. $1,2,3,4,5$ und 6$)\left(31^{*}\right)$

Die Einkristalle von 6d (aus Pentan $/ \mathrm{CH}_{2} \mathrm{Cl}_{2}(7 / 1)$ ), $\boldsymbol{E}$-11d $\boldsymbol{\alpha}$ (aus Pentan $/ \mathrm{CH}_{2} \mathrm{Cl}_{2}(8 / 2)$ ) und $\mathbf{1 2 d}$ (aus Pen$\left.\tan / \mathrm{CH}_{2} \mathrm{Cl}_{2}(8 / 2)\right)$ wurden bei Raumtemperatur in ein Markröhrchen eingeschmolzen. Die Datensammlung erfolgte in der Wyckoff-Messtechnik (Scanbreite: $4.0^{\circ}<2 \Theta<54.0^{\circ}$; Scangeschwindigkeit: variabel, $2.0^{\circ} / \mathrm{min}$ bis $29.3^{\circ} / \mathrm{min}$ ) mit einem Siemens R $3 \mathrm{~m} / \mathrm{V}$ Diffraktometer (Graphitmonochromator. Mo-K $\alpha$ Strahlung, $\lambda=0.71073 \AA$ ). Es wurde jeweils eine empirische Absorptionskorrektur (basierend auf 10 Reflexen) durchgeführt. Die Strukturen wurden mit Patterson-Methoden (Siemens SHFixTL-plus (VMS)) gelöst und nach dem Full-Matrix-Least-SquaresVerfahren verfeinert. Die Wasserstoffatome wurden in idealer Geometrie berechnet und als "Rigid Group" verfeinert.

\section{Dank}

Wir danken dem Fonds der Chemischen Industrie und dem Land Baden-Württemberg (Schwerpunkt- programm "Metallzentrierte Substrattransformationen") für die Förderung dieser Untersuchung, sowie Herrn B. Weibert für die Sammlung der Datensätze bei den Röntgenstrukturuntersuchungen.

\section{Literatur und Bemerkungen}

I E.O. Fischer. H.J. Kalder. A. Frank, F.H. Köhler und G. Huttner, Angew. Chem., 88 (1970) 683: Angew. Chem, Int Ed Engl, $15(1976) 623$.

2 H. Berke, Angew. Chem. \&s (1976) 684: Ansew. Chem. Int Ed. Engl, 15 (1976) 624.

3 Übersicht: (a) M.I. Bruce und A.G. Swincer. Ad Organomet. Chem., 22 (1983) 59: (b) M.1. Bruce, Chem. Ret., 91 (1991) 197.

$4 \mathrm{H}$. Berke. G. Huthner und J. \&. Severl. Z. Vaturforsch. Teil B. 36 (1981) 1277

5 (a) H. Le Bozec, K. Ouzzine und P.H. Dixneuf, I. Chem. Soc, Chem. Commun. (1989) 219; (b) H. Le Bozec. D. Pilette und P. Dixneuf, Nes: J. Chem. 1t(1990) 793 .

$6 \mathrm{H}$. Berke, G. Hutwer und J. v. Seyer, J. Organomet. Chem. 218 (1981) 193

7 N. Pirio, D. Touchard. L. Touper und P.IH. Dixneuf, J. Chem. Soc. Chem. Commut.. (1991)981).

8 H. Berke, P. Härter. G. Huttner und I. Zsolnat, Z. Nuturforsch, Teil B, 36 (1981) 929 .

9 V.N. Kalinin. V.V. Derunov. M.A. Lusenkova, P.V. Petrovsk und N.E. Kolobova. J. Organomet. Chem.. 370 (1989) 303.

10 N.E Kolobova, L.L. Janov, O.S. Zhvanko, O.M. Khitrova, A.S Batsanov and Yu.T. Struchkov. J. Organomet. (hem. 265 (1984) 271.

11 Berke at $u l$ erwähnen in [H], dass HNMe, ännlich wic 
$\left[\mathrm{NMe}_{2}\right]^{-} / \mathrm{H}^{+}$mit $\left(\eta^{5}-\mathrm{C}_{5} \mathrm{H}_{4} \mathrm{Me}\right)(\mathrm{CO})_{2} \mathrm{Mn}=\mathrm{C}=\mathrm{C}=\mathrm{CPh}_{2}$ zu einem Aminocarbenkomplex reagiert.

12 J.P. Selegue, Organometallics, 1 (1982) 217.

13 K.H. Dötz, Chem. Ber., 110 (1977) 78.

14 E. Moser und E.O. Fischer, J. Organomet. Chem., 16 (1969) 275.

15 A. Wienand, H.-U. Reissig, H. Fischer und J. Hofmann, Chem. Ber., 122 (1989) 1589.

16 z. B. (a) H. Fischer, U. Schubert und R. Märkl, Chem. Ber., 114 (1981) 3412; (b) H. Fischer, S. Zeuner, K. Ackermann und U. Schubert, J. Organomet. Chem., 263 (1984) 201; (c) F. Seitz, H. Fischer und J. Riede, J. Organomet. Chem., 287 (1985) 87; (d) D.C. Yang, V. Dragisich, W.D. Wulff und J.C. Huffmann, J. Am. Chem. Soc., 110 (1988) 307; (e) R. Aumann, S. Althaus, C. Krüger und P. Betz, Chem. Ber., 122 (1989) 357.

17 A.G.M. Barrett, C.P. Brock und M.A. Sturgess, Organometallics, 4 (1985) 1903

18 A.G.M. Barrett, J. Mortier, M. Sabat und M.A. Sturgess, Organometallics, 7 (1988) 2553.

19 E.O. Fischer und R. Aumann, Chem. Ber., 101 (1968) 963.

20 Y. Ito, T. Hirao und T. Saegusa, J. Organomet. Chem., 131 (1977) 121.
21 C.P. Casey und R.L. Anderson, J. Am. Chem. Soc., 96 (1974) 1230.

22 A.G.M. Barrett, N.E. Carpenter und M. Sabat, J. Organomet. Chem., 352 (1988) C8.

23 C.C. Costain und B.P. Stoicheff, J. Chem. Phys., 30 (1959) 777.

24 M.M. Midland, J. Org. Chem., 40 (1975) 2250.

25 P.L Pickard und T.L. Tolbert, Org. Synth., Coll. Vol., 5 (1973) 520.

26 P.L Pickard und D.J. Vaughan, J. Am. Chem. Soc., 72 (1950) 876.

27 H. Zaunschirm, Liebigs Ann. Chem., 245 (1888) 279.

28 C.H. Schmidt, Chem. Ber., 103 (1970) 986.

29 H. Fischer und S. Zeuner, J. Organomet. Chem., 286 (1985) 201.

30 D.W. Macomber und P. Madhukar, Organometallics, 8 (1989) 1275.

31 Weitere Einzelheiten zu den Kristallstrukturanalysen können beim Fachinformationszentrum Karlsruhe, Gesellschaft für wissenschaftlich-technische Information $\mathrm{mbH}, \mathrm{W}-7514$ EggensteinLeopoldshafen 2, unter Angabe der Hinterlegungsnummer CSD56846, der Autoren und des Zeitschriftenzitats angefordert werden. 